\title{
Condensation of $N$ bosons. II. Nonequilibrium analysis of an ideal Bose gas and the laser phase-transition analogy
}

\author{
V. V. Kocharovsky, ${ }^{1,3}$ Marlan O. Scully, ${ }^{1,2}$ Shi-Yao Zhu, ${ }^{1,4}$ and M. Suhail Zubairy ${ }^{1,5}$ \\ ${ }^{1}$ Department of Physics and Institute for Quantum Studies, Texas A\&M University, College Station, Texas 77843 \\ ${ }^{2}$ Max-Planck Institut fur Quantenoptik, 85748 Garching, Germany \\ ${ }^{3}$ Institute of Applied Physics of the Russian Academy of Science, Nizhny Novgorod, Russia \\ ${ }^{4}$ Department of Physics, Hong Kong Baptist University, Kowloon Tong, Hong Kong \\ ${ }^{5}$ Department of Electronics, Quaid-i-Azam University, Islamabad, Pakistan
}

(Received 6 July 1999; published 20 January 2000)

\begin{abstract}
A nonequilibrium approach to the dynamics and statistics of the condensate of an ideal $N$-atom Bose gas cooling via interaction with a thermal reservoir using the canonical ensemble is developed. We derive simple analytical expressions for the canonical partition function and equilibrium distribution of the number of atoms in the ground state of a trap under different approximations, and compare them with exact numerical results. The $N$-particle constraint associated with the canonical ensemble is usually a burden. In the words of Kittel, "in the investigation of the Bose-Einstein... laws it is very inconvenient to impose the restriction that the number of particles in the subsystem shall be held constant." But in the present approach, based on the analogy between a second-order phase transition and laser threshold behavior, the $N$-particle constraint makes the problem easier. We emphasize that the present work provides another example of a case in which equilibrium (detailed balance) solutions to nonequilibrium equations of motion provide a useful supplementary approach to conventional statistical mechanics. We also discuss some dynamical and mesoscopic aspects of Bose-Einstein condensation. The conclusion is that the present analytical (but approximate) results, based on a nonequilibrium approach, are in excellent agreement with exact (but numerical) results. The present analysis has much in common with the quantum theory of the laser.
\end{abstract}

PACS number(s): 03.75.Fi, 05.30.Jp

\section{INTRODUCTION}

Bose-Einstein condensation (BEC) [1-3] is, and has long been, a fascinating subject in its own right, and has become even more interesting in light of successful experimental demonstrations of BEC in dilute ${ }^{4} \mathrm{He}$ [4] and ultracold atomic gases [5-8]. Furthermore the production of "coherent atomic beams," the so-called atom laser [9], and its relation to the conventional laser, is intriguing, as is the relation between the BEC phase transition and the quantum theory of the laser [10].

However, as noted by Uhlenbeck [1(c)], the physics of BEC is subtle with many pitfalls and surprises. In the following numbered paragraphs we cite a few such difficulties and indicate how the present work handles (or mishandles) these problems.

(1a) Uhlenbeck criticized Einstein's arguments concerning the implied singularity in the equation of state at $T_{c}$. Kahn and Uhlenbeck later [1(d)] pointed out that the "discussion of the condensate requires that the bulk limit be taken in which the number and volume are made infinite with the density, $N / V$ fixed.' But this leaves open the question of how best to think about and define $T_{c}$ for finite mesoscopic systems.

(1b) In the present paper (see Sec. VII), and in our earlier paper [11], which we refer to as CNB I, we show that it is useful to follow the lead of laser theory wherein the critical threshold inversion is defined as that for which gain equals loss. That is, in recent work we showed that we can think of and redefine the critical temperature as being the temperature at which the gain in condensate particle number (due to cooling) equals the loss (due to heating). This yields the usual value of $T_{c}$, i.e., $T_{c}=\hbar \omega(N / \zeta(3))^{1 / 3}$ for a weak harmonic trap. We also find the Ketterle-van Druten [12] modified critical temperature expression $\sum_{n, l, m}\left\{\left[\exp \hbar \omega(l+m+n) / T_{c}\right.\right.$ $-1]\}^{-1}=N$ when we go to stronger traps. It should be emphasized that this approach extends the critical temperature concept to the mesoscopic systems, involving say $10^{3}$ atoms, in a natural fashion.

(2a) Furthermore as Ziff, Uhlenbeck, and Kac [3] pointed out that " [When] the grand canonical properties for the ideal Bose gas are derived, it turns out that some of them differ from the corresponding canonical properties-even in the bulk limit!' and later on they say: "The differences that we have just found between some bulk properties in the canonical and the grand canonical ensembles are particularly striking because they represent infinite systems for which it is usually supposed that the ensembles are equivalent... In this section we investigate the grand canonical ensemble and show that it loses its validity for the ideal Bose gas in the condensed region.' Most recently Holthaus, Kalinowski, and Kristen (HKK) note that [13] "There is, however, one serious failure of the grand canonical ensemble. Grand canonical statistics predicts that the mean-square fluctuation $\left\langle\Delta n_{\nu}^{2}\right\rangle_{\mathrm{gc}}$ of the $\nu$ th single-particle level's occupation equals $\left\langle n_{\nu}\right\rangle_{\mathrm{gc}}\left(\left\langle n_{\nu}\right\rangle_{\mathrm{gc}}+1\right)$. Applied to the ground state $\nu=0$, this gives $\left\langle\Delta n_{0}^{2}\right\rangle_{\mathrm{gc}}=\left\langle n_{0}\right\rangle_{\mathrm{gc}}\left(\left\langle n_{0}\right\rangle_{\mathrm{gc}}+1\right)$ even when the temperature $T$ approaches zero, so that all $N$ particles condense into the ground state. But the implication of huge fluctuations, $\left\langle\Delta n_{0}^{2}\right\rangle_{\mathrm{gc}}=N(N+1)$, is clearly unacceptable; when all par- 


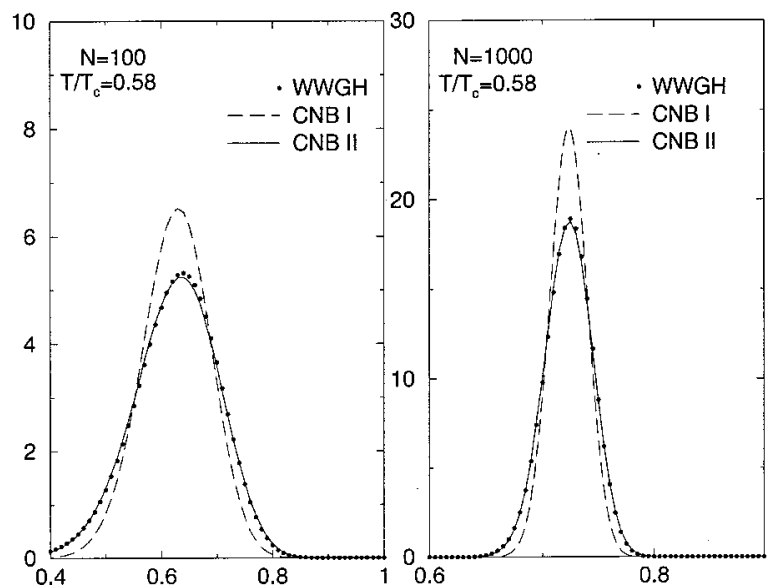

FIG. 1. Probability of having $n_{0}$ bosons in the ground state of a 3D harmonic trap. The dashed line is the approximation presented in CNB I. The solid line is the improved distribution of the present paper, CNB II. The dots are numerical results per WWGH. The vertical axis is $N p_{n_{0}}$, and the horizontal axis $n_{0} / N$ for both graphs.

ticles occupy the ground state, the fluctuation has to die out. This grand canonical fluctuation catastrophe has been discussed by generations of physicists, ... .',

(2b) We take a different approach to the problem, always working in the canonical ensemble [14]. Our results are in excellent agreement with exact numerical "experiments" of Wilkens and Weiss [15] and Grossmann and Holthaus [16], whose results are referred to as WWGH. In Fig. 1, we present the comparison of the results of CNB I with the exact results for the probability $p\left(n_{0}\right)$ of finding $n_{0}$ atoms in the ground state. The present paper focuses on the ideal noninteracting Bose gas. However the problem of anomalous fluctuations in the interacting gas is of current interest and is discussed in CNB III [18]. There we regain and extend the recent results of Giorgini, Pitaevskii and Stringari [17].

(3a) It is textbook wisdom that the canonical ensemble is not user friendly, to quote Kittel [19]: “In the investigation of the Bose-Einstein... laws it is very inconvenient to impose the restriction that the number of particles in the subsystem shall be held constant.",

(3b) However, in the present approach the $N$-particle constraint associated with the canonical ensemble makes the problem easier. The following quote from CNB I makes the point: "The $N$ particle constraint is included naturally in the present formulation... . We emphasize that the present work provides another example in which steady state (detailed balance) solutions to nonequilibrium equations of motion provide a supplementary approach to conventional statistical mechanics (e.g., partition function calculations). This is of interest since, for example, the partition sums in the canonical ensemble are complicated by the restriction to $N$ particles. Stated differently, the present approach lends itself to different approximations, yielding, among other things, a simple (approximate) analytic expression for the ground state density matrix for $N$ trapped bosons."

(4a) Herzog and Olshanii [20] noted that there was no known simple analytical expression for the canonical partition function. (4b) We presented a simple expression for $Z_{N}$ in CNB I, the present refined version of which is given by Eq. (7) below. We also point to the paper of HKK in this context. This important work was not known to us at the time of publication of CNB I. Detailed comparison between their work and ours will be given in a later paper.

We next turn to a discussion of our main results and their physical interpretation. As mentioned earlier, the condensation of bosonic atoms in a trap has obvious similarities to the threshold behavior of a laser. We recall that, in the quantum theory of laser, the dynamics of laser light is conveniently described by a master equation obtained by treating the atomic (gain) media and cavity dissipation (loss) as reservoirs which when "traced over' yield the coarse-grained equation of motion for the reduced density matrix for laser radiation. In this way we arrive at the equation of motion for the probability of having $n$ photons in the cavity [10]:

$$
\begin{aligned}
\dot{p}_{n}= & -\left[\frac{A}{1+B(n+1) / A}\right](n+1) p_{n}+\left[\frac{A}{1+B n / A}\right] n p_{n-1} \\
& -C n p_{n}+C(n+1) p_{n+1},
\end{aligned}
$$

where $A$ is the linear gain, $B$ is the nonlinear saturation which comes from the fact that after emission the atoms become "absorbers," and $C$ is the cavity decay rate. Solving Eq. (1) in the steady state we find

$$
p_{n}=\mathcal{N} \frac{(A / B) !\left(A^{2} / B C\right)^{n}}{(n+A / B) !}
$$

where the normalization can be written in terms of confluent hypergeometric functions as

$$
\mathcal{N}^{-1}=F\left(1, \frac{A}{B}+1, \frac{A^{2}}{B C}\right) .
$$

From Eq. (2) we have the important result that partially coherent laser light has a sharp photon distribution (with a width several times Poissonian for a typical He-Ne laser) due to the presence of $B$ in our equation. Thus we see that the saturation nonlinearity in the radiation matter interaction is essential for laser coherence.

One naturally has to ask whether the corresponding nonlinearity in BEC is due solely to atom-atom scattering, or if there is a nonlinearity even in an ideal Bose gas. As in CNB $\mathrm{I}$, we shall see that the latter is the case. More generally we pose the following question: Is there a similar nonequilibrium approach for BEC in a dilute atomic gas that helps us in understanding the underlying physical mechanisms for the condensation, the critical behavior, and the associated nonlinearities? The answer to this question, as discussed in CNB $\mathrm{I}$, is "yes" [11]. The extended and improved treatment is the subject of this paper.

Thus we consider the cooling of an ideal noninteracting $\mathrm{N}$-atom Bose gas confined inside a trap. The gas interacts with a thermal reservoir at temperature $T$. As pointed out above, most studies relating to this problem have been concerned with the evaluation of the partition function. Of course, the statistics of the Bose atoms in the ground state of 
a trap can be inferred from these partition functions. In general, there is a dearth of analytic expressions for the canonical partition function for the ideal Bose gas. However, thanks to WWGH, exact numerical results can be and have been obtained.

As discussed in CNB I and further explained in Sec. II of the present paper, we obtain a simple master equation for the density matrix of the Bose gas as it cools toward the ground state via heat exchange with a harmonic-oscillator reservoir. The master equation for the distribution function of the condensed bosons $p_{n_{0}}$ takes the form

$$
\begin{aligned}
\dot{p}_{n_{0}}= & -\kappa\left\{K_{n_{0}}\left(n_{0}+1\right) p_{n_{0}}-K_{n_{0}-1} n_{0} p_{n_{0}-1}\right. \\
& \left.+H_{n_{0}} n_{0} p_{n_{0}}-H_{n_{0}+1}\left(n_{0}+1\right) p_{n_{0}+1}\right\}
\end{aligned}
$$

and is seen to be similar to Eq. (1) for the laser. Here $\kappa H_{n_{0}}$ and $\kappa K_{n_{0}}$ are the heating and cooling coefficients with analogy to the cavity loss and saturated gain parameters in the laser master equation. These coefficients depend upon trap parameters such as the shape of the trap, the total number of bosons in the trap, $N$, and the temperature $T$.

Simple analytic expressions for $H_{n_{0}}$ and $K_{n_{0}}$ can be derived under various approximations and for various trap shapes. In CNB I, simple closed form expressions were derived for these coefficients for a three-dimensional (3D) harmonic trap. These expressions yield good qualitative agreement with the exact results, and yield insight into, for example, the meaning of the "critical temperature" for a mesoscopic system; see Sec. VII. In the weak trap limit Eq. (4) is given in CNB I as

$$
\begin{aligned}
\frac{1}{\kappa} \dot{p}_{n_{0}}= & -\left[(N+1)\left(n_{0}+1\right)-(n+1)^{2}\right] p_{n_{0}} \\
& +\left[(N+1) n_{0}-n_{0}^{2}\right] p_{n_{0}-1} \\
& -\left(\frac{T}{T_{c}}\right)^{3} N\left[n_{0} p_{n_{0}}-\left(n_{0}+1\right) p_{n_{0}+1}\right],
\end{aligned}
$$

where $T$ is the temperature of the heat bath and $T_{c}$ is the transition temperature. The physical meaning of the terms in Eq. (4) as flow of probability is given in Fig. 2.

Upon relaxing the restriction to a weak trap the "heating rate" term $\left(T / T_{c}\right)^{3} N$ in Eq. (5) is replaced by

$$
\mathcal{H}=\sum_{\{n\} \neq 0} \frac{1}{e^{\beta \epsilon(\{n\})}-1},
$$

where $\beta=1 / T$ and $\{n\}$ denote the quantum numbers appropriate for the chosen trap potential, e.g., for a harmonic trap $\epsilon(\{n\})=\hbar \omega(n+l+m)$. In such a case, the steady-state solution to Eq. (5) may be written as

$$
p_{n_{0}}=\frac{1}{Z_{N}} \frac{\mathcal{H}^{N-n_{0}}}{\left(N-n_{0}\right) !} .
$$

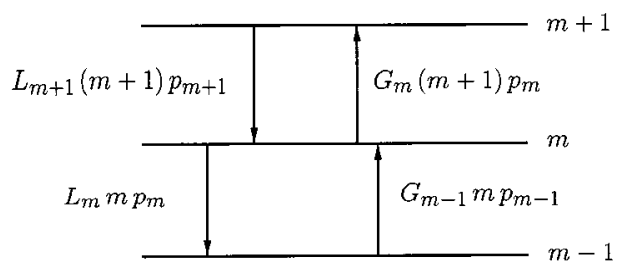

$\dot{p}_{m}=-G_{m}(m+1) p_{m}+G_{m-1} m p_{m-1}-L_{m} m p_{m}+L_{m+1}(m+1) p_{m+1}$

FIG. 2. Detailed balance equation and the corresponding probability flow diagram.

As presented in Fig. 3, here we extend CNB I and obtain improved analytical results for the distribution function of the condensed bosons, and hence the partition function. As shown in Sec. IV, Eq. (5) can be extended and improved in a natural way; see Fig. 3. We then find the following expressions for the condensate distribution $p_{n_{0}}$ and the partition function $Z_{N}$ :

$$
\begin{aligned}
& p_{n_{0}}=\frac{1}{Z_{N}} \frac{\left(N-n_{0}+\mathcal{H} / \eta-1\right) !}{(\mathcal{H} / \eta-1) !\left(N-n_{0}\right) !}\left(\frac{\eta}{1+\eta}\right)^{N-n_{0}}, \\
& Z_{N}=\sum_{n_{0}=0}^{N}\left(\begin{array}{c}
N-n_{0}+\mathcal{H} / \eta-1 \\
N-n_{0}
\end{array}\right)\left(\frac{\eta}{1+\eta}\right)^{N-n_{0}},
\end{aligned}
$$

where $\left(\begin{array}{l}r \\ s\end{array}\right)=r ! / s !(r-s) !$. The parameters $\mathcal{H}$ and $\eta$ are given for different trap potentials in Section V; in particular, for the harmonic trap,

$$
\eta=\mathcal{H}^{-1} \sum_{\{n\} \neq 0} \frac{1}{\left(e^{\left.\beta \hbar \Omega_{\{n\}}-1\right)^{2}} .\right.}
$$

This "approximate'" result is in excellent agreement with the exact numerical results for a wide range of parameters.

In Fig. 4-6, we present numerical comparison of the exact results for the distribution of condensed atoms, $p_{n_{0}}$, with these approximate explicit formulas in the particular case of three-dimensional isotropic harmonic trap (see Sec. V A) for various temperatures. The results indicate an excellent agreement between the exact results and the results based on quasithermal approximation. We also plot the mean value $\left\langle n_{0}\right\rangle$ (Fig. 7) and the variance squared $\Delta n_{0}^{2}$ (Fig. 8). In all cases the present approximate analytical results agree almost perfectly with the exact numerical ones.

The master equation approach of this paper also yields analytic results for the partition function, critical temperature, and distribution function for the condensed bosons for various shapes of the trap potential. Although we confine ourselves to a noninteracting Bose gas, the master equation approach provides a potentially powerful tool for studying BEC in systems with interacting gases as well. This will be further discussed elsewhere.

The paper is organized as follows. In Sec. II we present the "ideal gas plus reservoir" model, and outline the master equation approach to the description of the condensate in the canonical ensemble. We also present an explicit expression for the canonical partition function. In Sec. III we give the 


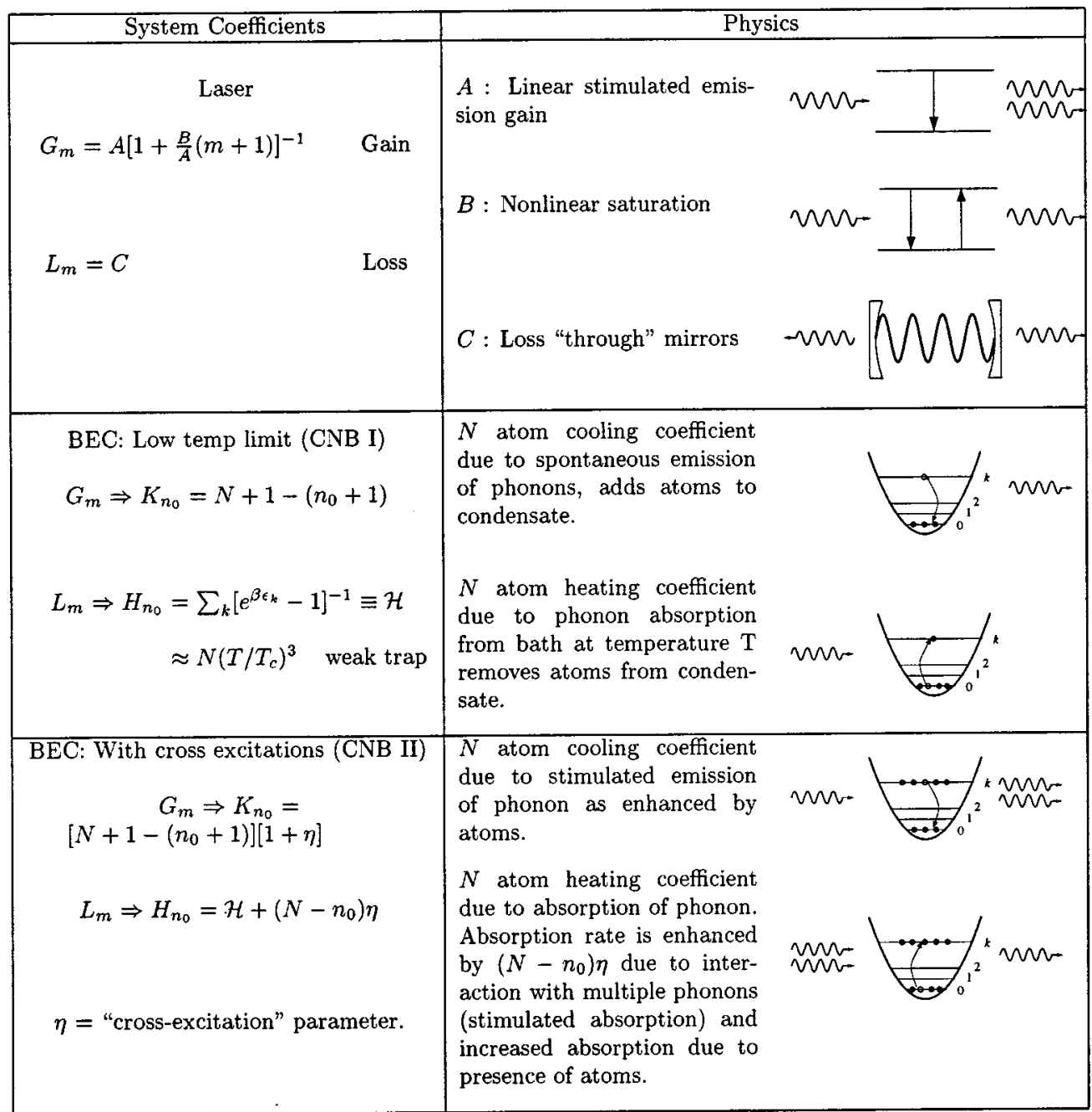

FIG. 3. Physical interpretation of various coefficients in the master equations.

steady-state solution to the master equation in the simplest low-temperature approximation, and in Sec. IV in a more complete and accurate quasithermal approximation. Main parameters of the different traps that determine statistics of the BEC are presented in Sec. V. Condensate statistics in the thermodynamic limit is summarized in Sec. VI. Dynamics and mesoscopic effects in BEC are discussed in Sec. VII. In Sec. VIII, we discuss the prospects for further applications of the master equation approach in the BEC problem.

\section{MASTER EQUATION FOR AN IDEAL BOSE GAS}

We consider the usual model of a dilute gas of Bose atoms when interatomic scattering is neglected. This ideal Bose gas is confined inside a trap, so that the number of atoms, $N$, is fixed but the total energy, $E$, of the gas is not fixed. Instead, the Bose atoms exchange energy with a reservoir which has a fixed temperature $T$. This "ideal gas plus reservoir' model allows us to demonstrate most clearly the master equation approach to the analysis of dynamics and statistics of BEC, and, in particular, the advantages and typical mathematical tools of the method. Its extension for the case of an interacting gas which includes usual many-body effects due to interatomic scattering will be discussed elsewhere.

Thus we study the so-called canonical ensemble problem. It describes, in some sense, an intermediate situation as compared with the microcanonical ensemble and the grandcanonical ensemble. In the microcanonical ensemble, the gas is completely isolated, $E=$ const and $N=$ const, so that there is no exchange of energy or atoms with a reservoir. In the grand-canonical ensemble, only the average energy per atom, i.e., the temperature $T$ and the average number of atoms $\langle N\rangle$ are fixed. In such a case there is an exchange of both energy and atoms with the reservoir.

The ideal gas plus thermal reservoir model provides the simplest description of many qualitative and, in some cases, quantitative characteristics of the BEC. In particular, it explains many features of the condensate dynamics and fluctuations. In the present paper, we shall focus mainly on the latter problem. The predictions of the grand-canonical ensemble model are not relevant in this respect to the actual BEC properties (see, e.g., the nice review in Ref. [3]). 

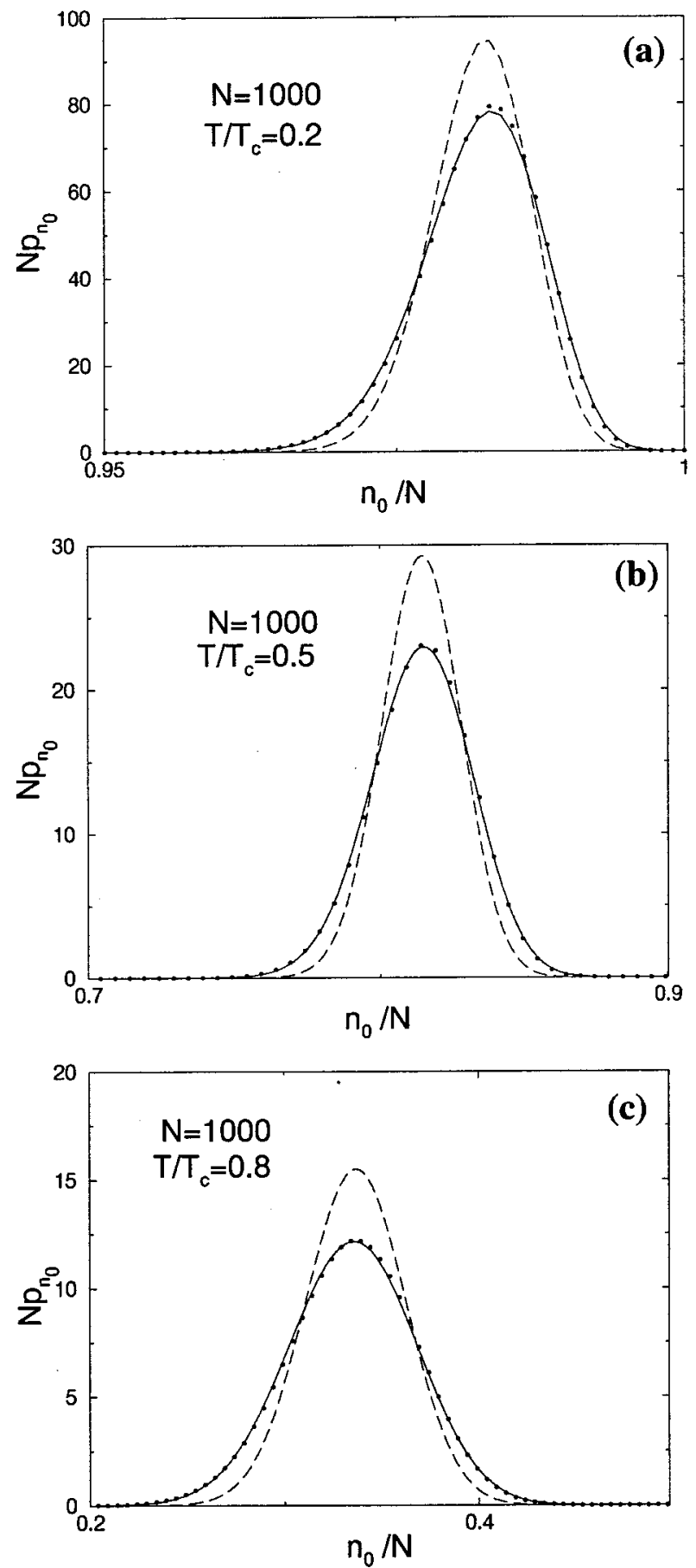

FIG. 4. The distributions of the number of atoms in the ground level of a trap, $p_{n_{0}}$, for different approximations [dashed lines for the low-temperature approximation (30), solid lines for the quasithermal approximation (40), and dots for the numerical simulation of the exact relations (44) and (46)] at different temperatures for a harmonic isotropic trap with $N=1000$.

\section{A. "Ideal gas plus thermal reservoir" model}

For many problems a concrete realization of the reservoir system is not very important if its energy spectrum is dense and flat enough. For the sake of simplicity, we assume that the reservoir is an ensemble of simple harmonic oscillators
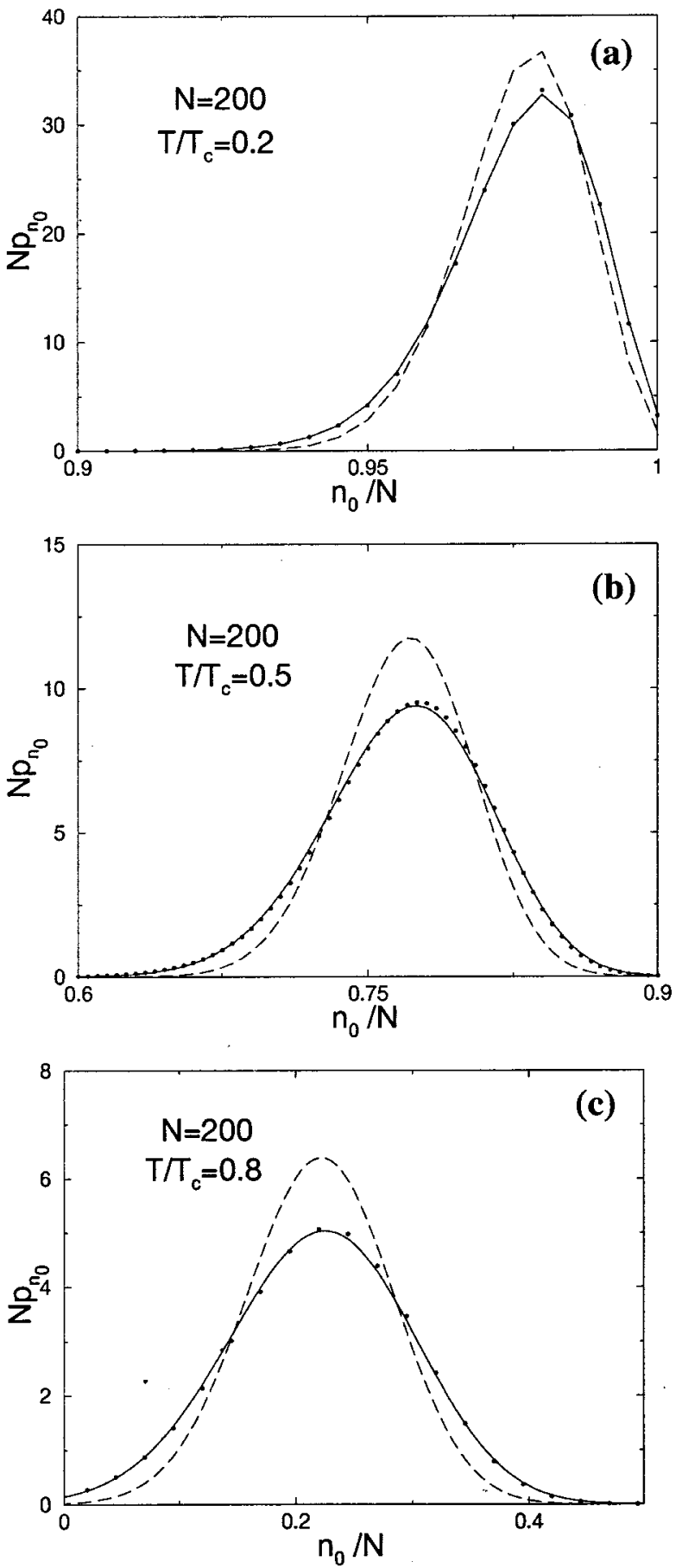

FIG. 5. The same as in Fig. 1 for a harmonic isotropic trap with $N=200$ atoms.

whose spectrum is dense and smooth enough. The interaction between the gas and the reservoir is described by the interaction picture Hamiltonian

$$
V=\sum_{j} \sum_{k>l} g_{j, k l} b_{j}^{\dagger} a_{k} a_{l}^{\dagger} e^{-i\left(\omega_{j}-\nu_{k}+\nu_{l}\right) t}+\text { H.c., }
$$

where $b_{j}^{\dagger}$ is the creation operator for the reservoir $j$ oscillator, 

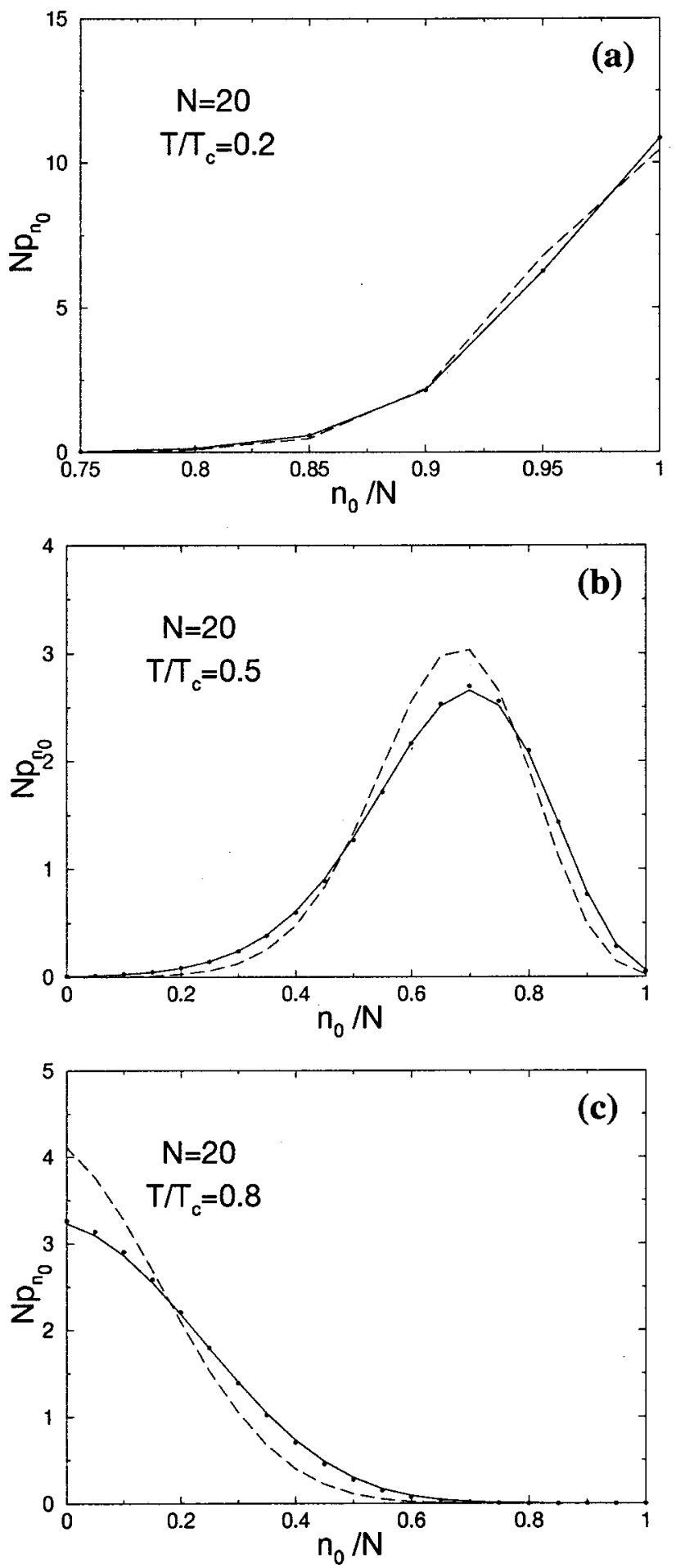

FIG. 6. The same as in Fig. 1 for a harmonic isotropic trap with $N=20$ atoms.

and $a_{k}^{\dagger}$ and $a_{k}(k \neq 0)$ are the creation and annihilation operators for the atoms in the $k$ th level. Here $\hbar \nu_{k}$ is the energy of the $k$ th level of the trap, and $g_{j, k l}$ is the coupling strength.

\section{B. Bose gas master equation}

The motion of the total gas plus reservoir system is governed by the equation for the total density matrix in the interaction representation,
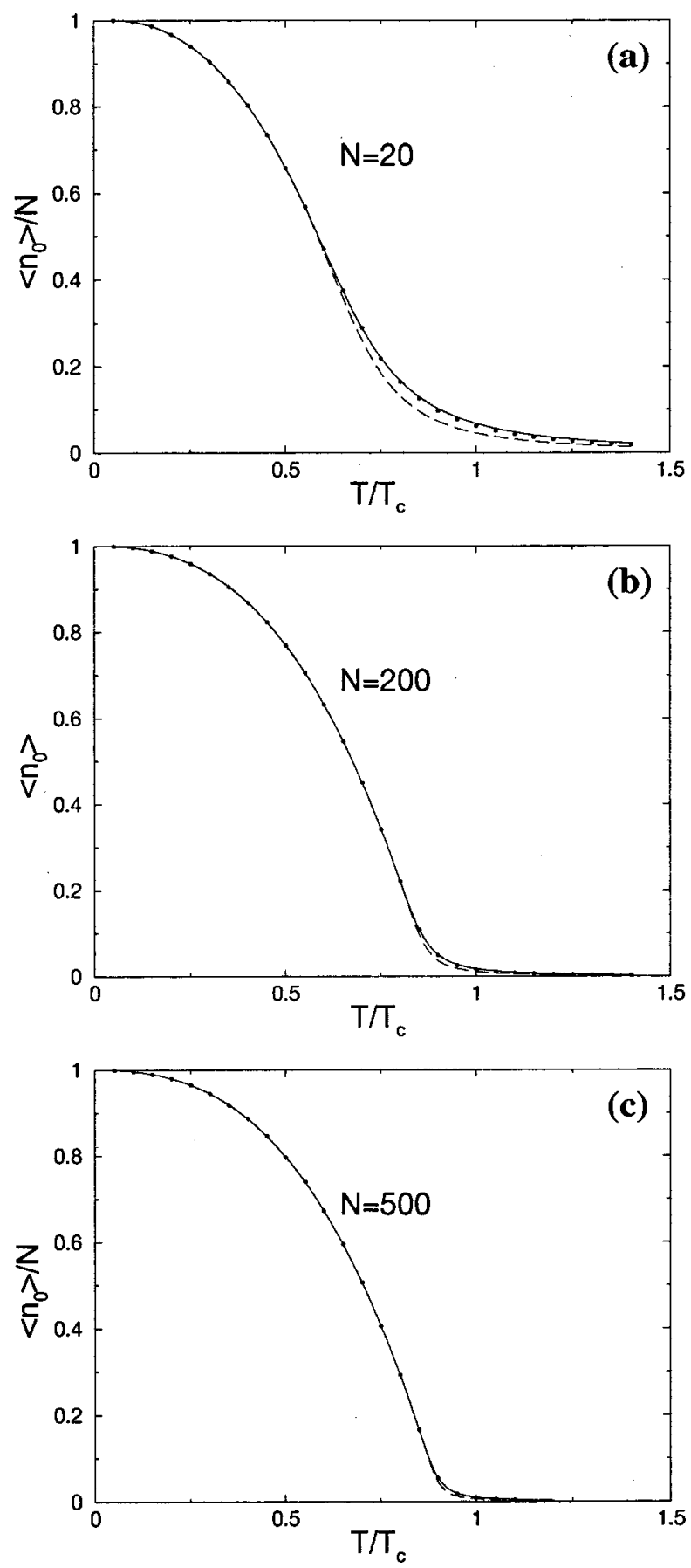

FIG. 7. The normalized average number of atoms in the ground level of a trap, $\left\langle n_{0}\right\rangle / N$, vs normalized temperature $T / T_{c}$, under various approximations [dashed lines for the low temperature approximation (30), solid lines for the quasithermal approximation (40), and dots for the numerical simulation of the exact relations (44) and (46)]. Harmonic isotropic trap with (a) $N=20$, (b) $N$ $=200$, and (c) $N=500$.

$$
\dot{\rho}_{\text {total }}(t)=-\frac{i}{\hbar}\left[V(t), \rho_{\text {total }}(t)\right]
$$

Integrating the above equation and inserting $\rho(t)$ into the commutator and tracing the reservoir, we obtain the exact 

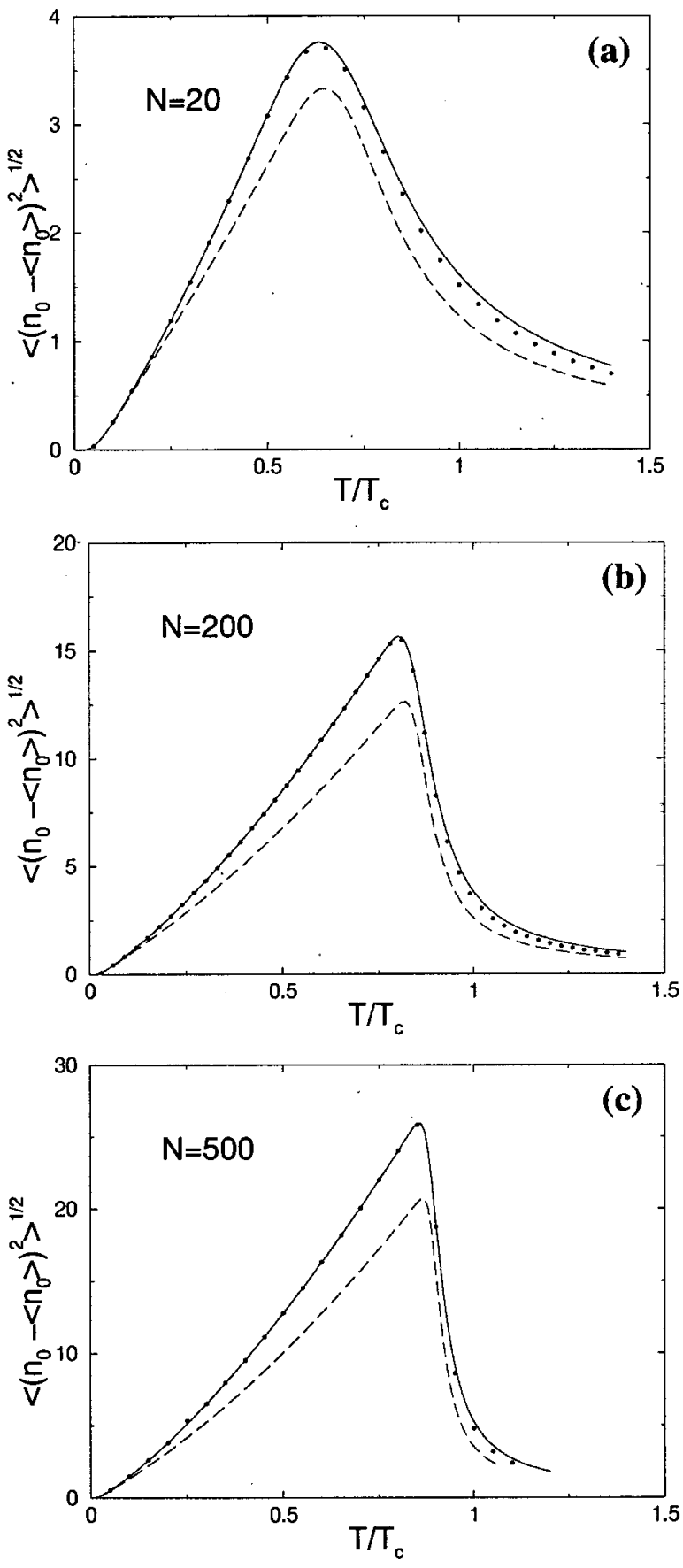

FIG. 8. The variance of the number of atoms in the ground level of a trap vs normalized temperature $T / T_{c}$, under various approximations [dashed lines for the low-temperature approximation (30), solid lines for the quasithermal approximation (40), and dots for the numerical simulation of the exact relations (44) and (46)]. Harmonic isotropic trap with (a) $N=20$, (b) $N=200$, and (c) $N=500$ atoms.

equation of motion for the density matrix of the Bose atoms subsystem,

$$
\dot{\rho}(t)=-\frac{1}{\hbar^{2}} \int_{0}^{t} d t^{\prime} \operatorname{Tr}_{\mathrm{res}}\left[V(t),\left[V\left(t^{\prime}\right), \rho_{\text {total }}\left(t^{\prime}\right)\right]\right],
$$

where $\operatorname{Tr}_{\text {res }}$ stands for the trace over the reservoir degrees of freedom. In Eq. (11) we omit the term $\operatorname{Tr}_{\text {res }}\left[V, \rho_{\text {total }}(t=0)\right]$, which is zero since $\rho_{\text {total }}(0)=\rho(0) \otimes \rho_{\text {res }}(0)$.

We assume that the reservoir is large and remains unchanged during the interaction with the dynamical subsystem (Bose gas). As is discussed in Appendix A, the density operator for the total system (gas plus reservoir) can then be factored, i.e., $\rho_{\text {total }}\left(t^{\prime}\right) \approx \rho\left(t^{\prime}\right) \otimes \rho_{\text {res }}$, where $\rho_{\text {res }}$ is the equilibrium density matrix of the reservoir. If the spectrum is smooth, we are justified in making the Markov approximation. We then obtain the following equation for the reduced density operator for the Bose subsystem:

$$
\begin{aligned}
\dot{\rho}= & -\frac{\kappa}{2} \sum_{k>l}\left(\eta_{k l}+1\right)\left[a_{k}^{\dagger} a_{l} a_{l}^{\dagger} a_{k} \rho\right. \\
& \left.-2 a_{l}^{\dagger} a_{k} \rho a_{k}^{\dagger} a_{l}+\rho a_{k}^{\dagger} a_{l} a_{l}^{\dagger} a_{k}\right] \\
& -\frac{\kappa}{2} \sum_{k>l} \eta_{k l}\left[a_{k} a_{l}^{\dagger} a_{l} a_{k}^{\dagger} \rho\right. \\
& \left.-2 a_{l} a_{k}^{\dagger} \rho a_{k} a_{l}^{\dagger}+\rho a_{k} a_{l}^{\dagger} a_{l} a_{k}^{\dagger}\right] .
\end{aligned}
$$

In deriving Eq. (12), we replaced the summation over reservoir modes by an integration with the density of reservoir modes $D\left(\omega_{k l}\right)$, and neglected the frequency dependence of the coefficient $\kappa=2 \pi D g^{2} / \hbar^{2}$; see Appendix A. Here

$$
\eta_{k l}=\eta\left(\omega_{k l}\right)=T r_{\mathrm{res}} b^{\dagger}\left(\omega_{k l}\right) b\left(\omega_{k l}\right)=\left[\exp \left(\hbar \omega_{k l} / T\right)-1\right]^{-1}
$$

is the average occupation number of the heat bath oscillator at frequency $\omega_{k l} \equiv \nu_{k}-\nu_{l}$. Equation (12) is the equation of motion for the Bose gas with a fixed number of atoms $N$.

\section{Condensate master equation}

What we are most interested in is the probability distribution

$$
p_{n_{0}}=\sum_{\left\{n_{k}\right\}_{n_{0}}} p_{n_{0},\left\{n_{k}\right\}_{n_{0}}}
$$

of the number of condensed atoms $n_{0}$, i.e., the number of atoms in the ground level of the trap. Here we introduce the probability of having $n_{0}$ atoms in the ground level and $n_{k}$ atoms in the $k$ th level,

$$
p_{n_{0},\left\{n_{k}\right\}_{n_{0}}}=\left\langle n_{0},\left\{n_{k}\right\}_{n_{0}}|\rho| n_{0},\left\{n_{k}\right\}_{n_{0}}\right\rangle,
$$

as a diagonal element of the density matrix in the canonical ensemble where $n_{0}+\Sigma_{k>0} n_{k}=N$ and $\left|n_{0},\left\{n_{k}\right\}_{n_{0}}\right\rangle$ is an arbitrary state of $N$ atoms with occupation numbers of the trap's energy levels, $n_{k}$, subject to the condition that there are $n_{0}$ atoms in the ground state of the trap.

In order to obtain an equation of motion for the condensate probability distribution $p_{n_{0}}$, we need to perform the summation over all possible occupations $\left\{n_{k}\right\}_{n_{0}}$ of the excited levels in the trap. The resulting equation of motion for $p_{n_{0}}$ is 


$$
\begin{aligned}
& \frac{d p_{n_{0}}}{d t}=-\kappa \sum_{\left\{n_{k}\right\}_{n_{0}}} \sum_{k>l>0} \\
& \times\left\{( \eta _ { k l } + 1 ) \left[\left(n_{l}+1\right) n_{k} p_{n_{0},\left\{n_{k}\right\}_{n_{0}}}\right.\right. \\
& \left.-n_{l}\left(n_{k}+1\right) p_{\left.n_{0},\left\{\ldots, n_{l}-1, \ldots, n_{k}+1, \ldots\right\}_{n_{0}}\right]}\right] \\
& +\eta_{k l}\left[n_{l}\left(n_{k}+1\right) p_{n_{0},\left\{n_{k}\right\}_{n_{0}}}\right.
\end{aligned}
$$

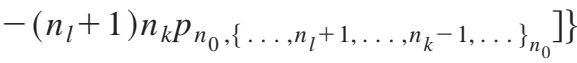

$$
\begin{aligned}
& -\kappa \sum_{\left\{n_{k}\right\}_{n_{0}}} \sum_{k^{\prime}>0}\left[\left(\eta_{k^{\prime}}+1\right)\left(n_{0}+1\right) n_{k^{\prime}} p_{n_{0},\left\{n_{k}\right\}_{n_{0}}}\right. \\
& -\left(\eta_{k^{\prime}}+1\right) n_{0}\left(n_{k^{\prime}}+1\right) p_{n_{0}-1,\left\{n_{k}+\delta_{k, k^{\prime}}\right\}_{n_{0}-1}} \\
& +\eta_{k^{\prime}} n_{0}\left(n_{k^{\prime}}+1\right) p_{n_{0},\left\{n_{k}\right\}_{n_{0}}}
\end{aligned}
$$

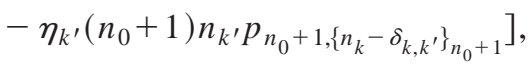

where $\eta_{k^{\prime}}=\eta\left(\nu_{k^{\prime}}\right)$ and the sum $\Sigma_{k^{\prime}}$ runs over all excited levels.

To simplify Eq. (16) we assume that the atoms in the excited levels with a given number of condensed atoms $n_{0}$ are in an equilibrium state at the temperature $T$, i.e.,

$$
p_{n_{0},\left\{n_{k}\right\}_{n_{0}}}=p_{n_{0}} \frac{\exp \left(-\frac{\hbar}{T} \sum_{k>0} \nu_{k} n_{k}\right)}{\sum_{\left\{n_{k}^{\prime}\right\}_{n_{0}}} \exp \left(-\frac{\hbar}{T} \sum_{k>0} \nu_{k} n_{k}^{\prime}\right)}
$$

where $\Sigma_{k>0} n_{k}=N-n_{0}$ and we assume that the sum $\Sigma_{k>0}$ runs over all energy states of the trap, including degenerate states whose occupations $n_{k}$ are treated as different stochastic variables. Equation (17) implies that the sum $\Sigma_{k>l>0}$ in Eq. (16) is equal to zero, since

$$
\begin{aligned}
& \left(\eta_{k l}+1\right) p_{n_{0},\left\{n_{k}\right\}_{n_{0}}}=\eta_{k l} p_{n_{0},\left\{\ldots, n_{l}+1, \ldots, n_{k}-1, \ldots\right\}_{n_{0}},}, \\
& \left(\eta_{k l}+1\right) p_{n_{0},\left\{\ldots, n_{l}-1, \ldots, n_{k}+1, \ldots\right\}_{n_{0}}}=\eta_{k l} p_{n_{0},\left\{n_{k}\right\}_{n_{0}}} .
\end{aligned}
$$

Equation (18) is precisely the detailed balance condition. The average number of atoms in an excited level, subject to the condition that there are $n_{0}$ atoms in the ground state, is equal to

$$
\left\langle n_{k^{\prime}}\right\rangle_{n_{0}}=\sum_{\left\{n_{k}\right\}_{n_{0}}} n_{k^{\prime}} \frac{p_{n_{0},\left\{n_{k}\right\}_{n_{0}}}}{p_{n_{0}}} .
$$

Therefore, the equation of motion for $p_{n_{0}}$ can be rewritten in the symmetrical and transparent form

$$
\begin{aligned}
\frac{d}{d t} p_{n_{0}}= & -\kappa\left\{K_{n_{0}}\left(n_{0}+1\right) p_{n_{0}}-K_{n_{0}-1} n_{0} p_{n_{0}-1}\right. \\
& \left.+H_{n_{0}} n_{0} p_{n_{0}}-H_{n_{0}+1}\left(n_{0}+1\right) p_{n_{0}+1}\right\},
\end{aligned}
$$

where

$$
\begin{aligned}
& K_{n_{0}}=\sum_{k^{\prime}>0}\left(\eta_{k^{\prime}}+1\right)\left\langle n_{k^{\prime}}\right\rangle_{n_{0}}, \\
& H_{n_{0}}=\sum_{k^{\prime}>0} \eta_{k^{\prime}}\left(\left\langle n_{k^{\prime}}\right\rangle_{n_{0}}+1\right) .
\end{aligned}
$$

We can obtain the steady state distribution of the number of atoms condensed in the ground level of the trap from Eq. (20). The mean value and the variance of the number of condensed atoms can then be determined. It is clear from Eq. (20) that there are two processes: cooling and heating. The cooling process is represented by the first two terms with the cooling coefficient $K_{n_{0}}$, and the heating by the third and fourth terms with the heating coefficient $H_{n_{0}}$. The detailed balance condition yields the following expression for the number distribution of the condensed atoms

$$
p_{n_{0}}=p_{0} \prod_{i=1}^{n_{0}} \frac{K_{i-1}}{H_{i}}
$$

Next we note that

$$
\begin{aligned}
& p_{n_{0},\left\{n_{k}\right\}_{n_{0}}} \\
& \quad=\frac{1}{Z_{N}}\left\langle n_{0},\left\{n_{k}\right\}_{n_{0}}\left|\exp \left(-\sum_{k} \epsilon_{k} \hat{n}_{k} / T\right)\right| n_{0},\left\{n_{k}\right\}_{n_{0}}\right),
\end{aligned}
$$

so that

$$
p_{n_{0}=N,\left\{n_{k}=0\right\}_{n_{0}}}=\frac{1}{Z_{N}} e^{-\epsilon_{0} N / T}=\frac{1}{Z_{N}} .
$$

In view of this we rewrite Eq. (23) as

$$
\begin{aligned}
p_{n_{0}} & =p_{0} \prod_{i=1}^{n_{0}} \frac{K_{i-1}}{H_{i}} \prod_{j=n_{0}+1}^{N} \frac{K_{j-1}}{H_{j}} \prod_{k=n_{0}+1}^{N} \frac{H_{k}}{K_{k-1}} \\
& =p_{N} \prod_{i=n_{0}+1}^{N} \frac{H_{i}}{K_{i-1}} .
\end{aligned}
$$

Hence the partition function $Z_{N}$ is determined by the normalization condition $\sum_{n_{0}=0}{ }^{N} p_{n_{0}}=1$, that is,

$$
Z_{N}=\frac{1}{p_{N}}=\sum_{n_{0}=0}^{N} \prod_{i=n_{0}+1}^{N}\left(\frac{K_{i-1}}{H_{i}}\right)^{-1} .
$$

The functions $H_{i}$ and $K_{i}$ as given by Eqs. (21) and (22) involve, along with $\eta_{k^{\prime}}$ [Eq. (13)], the function $\left\langle n_{k^{\prime}}\right\rangle_{n_{0}}$ [Eq. (19)]. In the following sections, we shall derive closed-form expressions for these quantities under various approximations. The master equation (20) for the distribution function 
for the condensed atoms is one of our main results. It yields explicit expressions for the statistics of the condensed atoms and the canonical partition function.

Under the above assumption of a thermal equilibrium for noncondensed atoms, we have

$$
\left\langle n_{k^{\prime}}\right\rangle_{n_{0}}=\frac{\sum_{\left\{n_{k}\right\}_{n_{0}}} n_{k^{\prime}} \exp \left(-\frac{\hbar}{T} \sum_{k>0} \nu_{k} n_{k}\right)}{\sum_{\left\{n_{k}^{\prime \prime}\right\}_{n_{0}}} \exp \left(-\frac{\hbar}{T} \sum_{k>0} \nu_{k} n_{k}^{\prime \prime}\right)} .
$$

In the next two sections we present different approximations that clarify general result (23).

\section{LOW-TEMPERATURE APPROXIMATION}

At low enough temperatures, the average occupations in the reservoir are small and $\eta_{k^{\prime}}+1 \simeq 1$ in Eq. (21). This suggests the simplest approximation for the cooling coefficient

$$
K_{n_{0}} \simeq \sum_{k}\left\langle n_{k}\right\rangle_{n_{0}}=N-n_{0}
$$

In addition, at very low temperatures the number of noncondensed atoms is also very small, we can therefore approximate $\left\langle n_{k^{\prime}}\right\rangle_{n_{0}}+1$ by 1 in Eq. (22). Then the heating coefficient is a constant equal to the total average number of thermal excitations in the reservoir at all energies corresponding to the energy levels of the trap:

$$
H_{n_{0}} \simeq \mathcal{H}, \quad \mathcal{H} \equiv \sum_{k>0} \eta_{k}=\sum_{k>0}\left(e^{\hbar \nu_{k} / T}-1\right)^{-1}
$$

Under these approximations, the condensate master equation (20) simplifies considerably, and contains only one nontrivial parameter $\mathcal{H}$. We obtain

$$
\begin{aligned}
\frac{d}{d t} p_{n_{0}}= & -\kappa\left\{\left(N-n_{0}\right)\left(n_{0}+1\right) p_{n_{0}}-\left(N-n_{0}+1\right) n_{0} p_{n_{0}-1}\right. \\
& \left.+\mathcal{H}\left[n_{0} p_{n_{0}}-\left(n_{0}+1\right) p_{n_{0}+1}\right]\right\} .
\end{aligned}
$$

It may be noted that Eq. (28) has the same form as the equation of motion for the photon distribution function in a laser operating not too far above threshold. The identification is complete if we define the gain, saturation, and loss parameters in the laser master equation by $\kappa(N+1), \kappa$, and $\kappa \mathcal{H}$, respectively. The mechanism for gain, saturation, and loss are however different in the present case.

A laser phase transition analogy exists via the $P$ representation $[21,22]$. A formal similarity with the laser equation leads to a Ginzburg-Landau-type free energy [10,21,22]

$$
G(I)=a(T) I+b(T) I^{2},
$$

where $a(T)=-(N-\mathcal{H}) / N$ and $b(T)=1 / 2 N$ for large $N$ near $T_{c}$.

The resulting steady-state distribution for the number of condensed atoms is given by

$$
p_{n_{0}}=\frac{1}{Z_{N}} \frac{\mathcal{H}^{N-n_{0}}}{\left(N-n_{0}\right) !}
$$

where $Z_{N}=1 / p_{N}$ is the partition function. It follows from the normalization condition $\sum_{n_{0}} p_{n_{0}}=1$ that

$$
Z_{N}=e^{\mathcal{H}} \Gamma(N+1, \mathcal{H}) / N !,
$$

where

$$
\Gamma(\alpha, x)=\int_{x}^{\infty} t^{\alpha-1} e^{-t} d t
$$

is an incomplete gamma function.

The distribution (30) can be presented as a probability distribution for the total number of noncondensed atoms, $n$ $=N-n_{0}$ :

$$
P_{n} \equiv p_{N-n}=\frac{e^{-\mathcal{H}} N !}{\Gamma(N+1, \mathcal{H})} \frac{\mathcal{H}^{n}}{n !}
$$

This looks something like a Poisson distribution; however, due to the additional normalization factor, $N ! / \Gamma(N+1, \mathcal{H})$ $\neq 1$, and a finite number of admissible values of $n$ $=0,1, \ldots, N$, it is not Poissonian. The mean value and the variance can be calculated from the distribution (30) for an arbitrary finite number of atoms in the Bose gas:

$$
\begin{gathered}
\left\langle n_{0}\right\rangle=N-\mathcal{H}+\mathcal{H}^{N+1} / Z_{N} N !, \\
\Delta n_{0}^{2} \equiv\left\langle n_{0}^{2}\right\rangle-\left\langle n_{0}\right\rangle^{2}=\mathcal{H}\left(1-\left(\left\langle n_{0}\right\rangle+1\right) \mathcal{H}^{N} / Z_{N} N !\right) .
\end{gathered}
$$

As we shall see from the extended treatment in Sec. IV, approximations (26) and (27) and therefore the results (33) and (34) are clearly valid at low temperatures, i.e., in the weak trap limit, $T \ll \varepsilon_{1}$, where $\varepsilon_{1}$ is an energy gap between the first excited and the ground levels of a single-particle spectrum in the trap. However, in the case of a harmonic trap, results (33) and (34) show qualitatively correct behavior for all temperatures, including $T \gg \varepsilon_{1}$ and $T \sim T_{c}$ [11].

\section{QUASITHERMAL APPROXIMATION FOR NONCONDENSATE OCCUPATIONS}

At arbitrary temperatures, a very reasonable approximation for the average noncondensate occupation numbers in the cooling and heating coefficients, [Eqs. (21) and (22)], is suggested by Eq. (25) in a quasithermal form,

$$
\left\langle n_{k}\right\rangle_{n_{0}}=\left(N-n_{0}\right) \eta_{k} / \sum_{k} \eta_{k}=\left(N-n_{0}\right) /\left[e^{\varepsilon_{k} / T}-1\right] \mathcal{H},
$$

where $\varepsilon_{k}=\hbar \nu_{k}, \eta_{k}$ is given by Eq. (13) and $\mathcal{H}$ by Eq. (27). Equation (35) satisfies the canonical ensemble constraint $N$ $=n_{0}+\sum_{k>0} n_{k}$ independently of the resulting distribution $p_{n_{0}}$. This important property is based on the fact that a quasithermal distribution (35) provides the same relative average occupations in excited levels of the trap as in the thermal reservoir [Eq. (13)]. 
In this approximation, the cooling and heating coefficients (21) and (22) are

$$
\begin{gathered}
K_{n_{0}}=\left(N-n_{0}\right)(1+\eta), \\
H_{n_{0}}=\mathcal{H}+\left(N-n_{0}\right) \eta .
\end{gathered}
$$

Compared with the low temperature approximations (26) and (27), these coefficients acquire an additional contribution $\left(N-n_{0}\right) \eta$ due to the cross-excitation parameter

$$
\eta=\frac{1}{N-n_{0}} \sum_{k>0}\left\langle\eta_{k}\right\rangle\left\langle n_{k}\right\rangle_{n_{0}}=\frac{1}{\mathcal{H}} \sum_{k>0} \frac{1}{\left(e^{\varepsilon_{k} / T}-1\right)^{2}} .
$$

Now the condensate master equation (20) contains two nontrivial parameters $\mathcal{H}$ and $\eta$ :

$$
\begin{aligned}
\frac{d p_{n_{0}}}{d t}= & -\kappa\left\{( 1 + \eta ) \left[\left(N-n_{0}\right)\left(n_{0}+1\right) p_{n_{0}}\right.\right. \\
& \left.-\left(N-n_{0}+1\right) n_{0} p_{n_{0}-1}\right]+\left[\mathcal{H}+\left(N-n_{0}\right) \eta\right] n_{0} p_{n_{0}} \\
& \left.-\left[\mathcal{H}+\left(N-n_{0}-1\right) \eta\right]\left(n_{0}+1\right) p_{n_{0}+1}\right\} .
\end{aligned}
$$

The steady-state solution of Eq. (39) is given by

$$
\begin{aligned}
p_{n_{0}} & =\frac{1}{Z_{N}} \frac{\left(N-n_{0}+\mathcal{H} / \eta-1\right) !}{(\mathcal{H} / \eta-1) !\left(N-n_{0}\right) !}\left(\frac{\eta}{1+\eta}\right)^{N-n_{0}} \\
& =\frac{1}{Z_{N}}\left(\begin{array}{c}
N-n_{0}+\frac{\mathcal{H}}{\eta}-1 \\
N-n_{0}
\end{array}\right)\left(\frac{\eta}{1+\eta}\right)^{N-n_{0}},
\end{aligned}
$$

where the canonical partition function $Z_{N}=1 / p_{N}$ is

$$
Z_{N}=\sum_{n_{0}=0}^{N}\left(\begin{array}{c}
N-n_{0}+\mathcal{H} / \eta-1 \\
N-n_{0}
\end{array}\right)\left(\frac{\eta}{1+\eta}\right)^{N-n_{0}} .
$$

The master equation (39) for $p_{n_{0}}$, and the analytic approximate expressions (40) and (41) for the condensate distribution function $p_{n_{0}}$ and the partition function $Z_{N}$, respectively, are among the main results of this paper. As we shall see later, they provide a very accurate description of the Bose gas for a very large range of parameters and for different trap potentials.

The canonical partition function (41) also allows us to calculate the microcanonical partition function $\Omega(E, N)$ by means of the inversion of the definition

$$
Z_{N}(T)=\sum_{E=0}^{\infty} e^{-E / T} \Omega(E ; N) .
$$

In particular, for the isotropic harmonic trap with an eigenfrequency $\omega$ this can be accomplished by an application of the saddle-point approximation to the contour integral:

$$
\Omega(E ; N)=\frac{1}{2 \pi i} \oint_{\gamma} \frac{Z_{N}}{x^{1+E / \hbar \omega}} d x, \quad x=e^{-\hbar \omega / T} .
$$

An accurate knowledge of the canonical partition function is essential for the calculation of the microcanonical condensate fluctuations by the saddle-point method, as was demonstrated recently [16] by a numerical comparison with exact microcanonical simulations. If we start with the grandcanonical partition function and apply the saddle-point approximation twice, first to obtain the canonical partition function from the grand canonical one and then to invert Eq. (42) via Eq. (43), the result would be incorrect for $E$ $<N \hbar \omega_{1}$ where $\hbar \omega_{1}$ is the energy of the first excited state (see Refs. [16,23]). In principle, the knowledge of the canonical partition function allows us to calculate thermodynamic and statistical equilibrium properties of the system in the standard way (see, e.g., Refs. [3,24]).

There is a general relation between the probability distribution of the number of atoms in the ground state, $p_{n_{0}}$, and the canonical partition function [15]

$$
p_{n_{0}}=\frac{Z_{N-n_{0}}(T)-Z_{N-n_{0}-1}(T)}{Z_{N}(T)},
$$

which is obviously satisfied by our explicit formula (40).

Previously, the closed-form expression for the canonical partition function (42) was known only for one-dimensional harmonic traps $[25,26]$ :

$$
Z_{N}(T)=\prod_{k=1}^{N} \frac{1}{1-e^{-k \hbar \omega / T}} .
$$

In the general case, there exists only a recursion relation that is quite complicated and difficult for analysis $[15,24,27,28]$ :

$$
Z_{N}(T)=\frac{1}{N} \sum_{k=1}^{N} Z_{1}(T / k) Z_{N-k}(T) .
$$

However, Eq. (46) can be used for numerical calculation of the condensate fluctuations via relation (44) for up to, say $N \sim 10^{5}$ atoms.

Distribution (40) can also be presented as a probability distribution for the total number of noncondensed atoms, $n$ $=N-n_{0}$ :

$$
P_{n}=p_{N-n}=\frac{1}{Z_{N}}\left(\begin{array}{c}
n+\mathcal{H} / \eta-1 \\
n
\end{array}\right)\left(\frac{\eta}{1+\eta}\right)^{n} .
$$

Distribution (47) has the form of the well-known negative binomial distribution [29],

$$
P_{n}=\left(\begin{array}{c}
n+M-1 \\
n
\end{array}\right) q^{n}(1-q)^{M}, \quad n=0,1,2, \ldots, \infty,
$$

that was so named due to a coincidence of the probabilities $P_{n}$ with the terms in the negative-power binomial formula

$$
\frac{1}{(1-q)^{M}}=\sum_{n=0}^{\infty}\left(\begin{array}{c}
n+M-1 \\
n
\end{array}\right) q^{n} .
$$


It has a semantic origin similar to that of the well-known binomial distribution $P_{n}=\left(\begin{array}{c}M \\ n\end{array}\right)(1-q)^{n} q^{M-n}$, which was named after a Newton's binomial formula $[q+(1-q)]^{M}$ $=\sum_{n=0}^{M}\left(\begin{array}{l}M \\ n\end{array}\right)(1-q)^{n} q^{M-n}$. Distribution (47) is a negative binomial distribution only in the limit $N \gg(1+\eta) \mathcal{H}$.

The average number of atoms condensed in the ground state of the trap is

$$
\left\langle n_{0}\right\rangle \equiv \sum_{n_{0}=0}^{N} n_{0} p_{n_{0}} .
$$

It follows, on substituting for $p_{n_{0}}$ from Eq. (40), that

$$
\left\langle n_{0}\right\rangle=N-\mathcal{H}+(N+\mathcal{H} / \eta) p_{0} .
$$

The centered moments of the $m$ th order, $m>1$, of the number-of-condensed-atom and number-of-noncondensedatom fluctuations are equal to each other for even orders, and have opposite signs for odd orders:

$$
\left\langle\left(n_{0}-\bar{n}_{0}\right)^{m}\right\rangle=(-1)^{m}\left\langle(n-\bar{n})^{m}\right\rangle .
$$

The squared variance can be represented as

$$
\Delta n_{0}^{2}=\left\langle n^{2}\right\rangle-\langle n\rangle^{2}=\sum_{n=0}^{N} n(n-1) P_{n}+\langle n\rangle-\langle n\rangle^{2},
$$

and calculated analytically. We obtain

$$
\begin{aligned}
\Delta n_{0}^{2}= & (1+\eta) \mathcal{H}-p_{0}(\eta N+\mathcal{H})\left(N+\eta+\frac{\mathcal{H}-1}{1+2 \eta}\right) \\
& -p_{0}^{2}(\eta N+\mathcal{H})^{2},
\end{aligned}
$$

where

$$
p_{0}=\frac{1}{Z_{N}} \frac{(N+\mathcal{H} / \eta-1) !}{N !(\mathcal{H} / \eta-1) !}\left(\frac{\eta}{1+\eta}\right)^{N}
$$

is the probability that there are no atoms in the condensate.

For the "condensed phase" in the thermodynamic limit, the probability $p_{0}$ vanishes exponentially if the temperature is not very close to the critical temperature. In this case only the first term in Eq. (54) remains, resulting in

$$
\Delta n_{0}^{2}=(1+\eta) \mathcal{H} \equiv \sum_{k>0}\left(\left\langle n_{k}\right\rangle^{2}+\left\langle n_{k}\right\rangle\right) .
$$

This result was obtained earlier by standard statistical methods (see Ref. [3] and references therein).

It is easy to see that result (47) reduces to the simple approximation (32) in the formal limit $\eta \rightarrow 0, \mathcal{H} / \eta \rightarrow \infty$, when

$$
\frac{\Gamma\left(N-n_{0}+\mathcal{H} / \eta\right)}{\Gamma(N+\mathcal{H} / \eta)} \rightarrow\left(\frac{\mathcal{H}}{\eta}\right)^{-n_{0}}
$$

The limit applies to only very low temperatures, $T \ll \varepsilon_{1}$. However, due to Eqs. (27) and (38), the parameter $\mathcal{H} / \eta$ tends to 1 as $T \rightarrow 0$, but never to infinity. Nevertheless, re- sults (51) and (54) agree with the low-temperature approximation results (33) and (34) for $T \ll \varepsilon_{1}$. In this case the variance $\sqrt{\Delta n_{0}^{2}}$ is determined mainly by a square root of the mean value $\langle n\rangle$, which is correctly approximated by Eq. (33) as $\langle n\rangle \equiv N-\left\langle n_{0}\right\rangle \approx \mathcal{H}$.

\section{MAIN PARAMETERS OF DIFFERENT TRAPS}

As we have seen, the condensate fluctuations are governed mainly by two parameters: the number of thermal excitations $\mathcal{H}$ and the cross-excitation parameter $\eta$. They are determined by a single-particle energy spectrum of the trap. We explicitly present them below for different traps. We discuss mainly the three-dimensional case. A generalization to other dimensions is straightforward and is given in Sec. VC.

\section{A. Harmonic asymmetric trap}

The potential in the harmonic trap has, in general, an asymmetrical profile in space,

$$
V_{\mathrm{ext}}(x, y, z)=\frac{m}{2}\left(x^{2} \omega_{x}^{2}+y^{2} \omega_{y}^{2}+z^{2} \omega_{z}^{2}\right),
$$

with eigenfrequencies $\left\{\omega_{x}, \omega_{y}, \omega_{z}\right\}=\omega, \omega_{z} \geqslant \omega_{y} \geqslant \omega_{x}>0$. Here $m$ is the mass of the atom. The single-particle energy spectrum of the trap,

$$
\varepsilon_{\mathbf{k}}=\hbar \mathbf{k} \omega \equiv \hbar\left(k_{x} \omega_{x}+k_{y} \omega_{y}+k_{z} \omega_{z}\right),
$$

can be enumerated by three non-negative integers $\left\{k_{x}, k_{y}, k_{z}\right\}=\mathbf{k}, k_{x, y, z} \geqslant 0$. We have

$$
\begin{gathered}
\mathcal{H}=\sum_{\mathbf{k}>0} \frac{1}{e^{\hbar \mathbf{k} / T}-1}, \\
\eta \mathcal{H}=\sum_{\mathbf{k}>0} \frac{1}{\left(e^{\hbar \mathbf{k} / T}-1\right)^{2}} .
\end{gathered}
$$

The energy gap between the ground state and the first excited state in the trap is equal to $\varepsilon_{1}=\hbar \omega_{x}$.

If the sums can be replaced by the integrals (continuum approximation), i.e., if $\hbar \omega_{x} \ll T$, the parameters $\mathcal{H}$ and $\eta \mathcal{H}$ are equal to (see Appendix B)

$$
\begin{gathered}
\mathcal{H}=\frac{T^{3}}{\hbar^{3} \omega_{x} \omega_{y} \omega_{z}} \zeta(3)=\left(\frac{T}{T_{c}}\right)^{3} N \\
\eta \mathcal{H}=\frac{T^{3}}{\hbar^{3} \omega_{x} \omega_{y} \omega_{z}}(\zeta(2)-\zeta(3))=\left(\frac{T}{T_{c}}\right)^{3} N \frac{\zeta(2)-\zeta(3)}{\zeta(3)},
\end{gathered}
$$

where a standard critical temperature is introduced as

$$
T_{c}=\hbar\left(\frac{\omega_{x} \omega_{y} \omega_{z} N}{\zeta(3)}\right)^{1 / 3}, \quad \zeta(3)=1.202 \ldots, \quad \zeta(2)=\frac{\pi^{2}}{6} .
$$


Therefore, the cross-excitation parameter $\eta$ is a constant, independent of the temperature and the number of atoms, and is given by

$$
\eta=\frac{\zeta(2)-\zeta(3)}{\zeta(3)} \approx 0.37
$$

The ratio

$$
\frac{\mathcal{H}}{\eta}=\frac{\zeta(3)}{\zeta(2)-\zeta(3)}\left(\frac{T}{T_{c}}\right)^{3} N
$$

goes to infinity in the thermodynamic limit proportionally to the number of atoms $N$.

In the opposite case of very low temperatures, $T \ll \hbar \omega_{x}$, we have

$$
\begin{gathered}
\mathcal{H} \approx \exp \left(-\frac{\hbar \omega_{x}}{T}\right)+\exp \left(-\frac{\hbar \omega_{y}}{T}\right)+\exp \left(-\frac{\hbar \omega_{z}}{T}\right), \\
\eta \mathcal{H} \approx \exp \left(-\frac{2 \hbar \omega_{x}}{T}\right)+\exp \left(-\frac{2 \hbar \omega_{y}}{T}\right)+\exp \left(-\frac{2 \hbar \omega_{z}}{T}\right),
\end{gathered}
$$

with an exponentially good accuracy. Now the crossexcitation parameter $\eta$ depends exponentially on the temperature and, instead of the number 0.37 [Eq. (65)], is exponentially small. The ratio

$$
\frac{\mathcal{H}}{\eta}=\frac{\left[\exp \left(-\frac{\hbar \omega_{x}}{T}\right)+\exp \left(-\frac{\hbar \omega_{y}}{T}\right)+\exp \left(-\frac{\hbar \omega_{z}}{T}\right)\right]^{2}}{\exp \left(-\frac{2 \hbar \omega_{x}}{T}\right)+\exp \left(-\frac{2 \hbar \omega_{y}}{T}\right)+\exp \left(-\frac{2 \hbar \omega_{z}}{T}\right)} \sim 1
$$

becomes approximately a constant. The particular case of an isotropic harmonic trap is described by the same Eqs. (58)(69) if we substitute $\omega_{x}=\omega_{y}=\omega_{z}=\omega$.

\section{B. Box ("homogeneous gas")}

In a cubic box with a size $L$, the energy spectrum of a moving atom is similar to the spectrum of a free motion:

$$
\varepsilon_{\mathbf{k}}=\frac{2 \pi^{2} \mathbf{k}^{2} \hbar^{2}}{m L^{2}}, \quad \mathbf{k}=\left\{k_{x}, k_{y}, k_{z}\right\} .
$$

The difference is that an admissible momentum is quantized and is enumerated by three integers $-\infty<k_{i}<+\infty, i$ $=x, y$, and $z$. We then have

$$
\begin{gathered}
\mathcal{H}=\sum_{\mathbf{k} \neq 0} \frac{1}{e^{\varepsilon_{1} \mathbf{k}^{2} / T}-1}, \\
\eta \mathcal{H}=\sum_{\mathbf{k} \neq 0} \frac{1}{\left(e^{\varepsilon_{1} \mathbf{k}^{2} / T}-1\right)^{2}},
\end{gathered}
$$

where

$$
\varepsilon_{1}=\frac{2 \pi^{2} \hbar^{2}}{m L^{2}}
$$

is the energy gap between the ground state and the first excited state in the box.

Let us again consider first the case $\varepsilon_{1} \ll T$ and use the continuum approximation. In the box, contrary to the previous case of the harmonic trap, the sum can be replaced by the integral only for the parameter $\mathcal{H}$, Eq. (71), leading to

$$
\mathcal{H}=\frac{(2 m T)^{3 / 2} L^{3}}{4 \pi^{2} \hbar^{3}} \frac{\sqrt{\pi}}{2} \zeta\left(\frac{3}{2}\right)=\left(\frac{T}{T_{c}}\right)^{3 / 2} N
$$

where we introduced the critical temperature

$$
T_{c}=\frac{2 \pi \hbar^{2}}{(\zeta(3 / 2))^{2 / 3} m L^{2}} N^{2 / 3}=\frac{\varepsilon_{1}}{\pi(\zeta(3 / 2))^{2 / 3}} N^{2 / 3}
$$

The second parameter has a well-known formal infrared divergence $[3,17,30]$ and should be calculated via the discrete sum (see Appendix B),

$$
\eta \mathcal{H}=S_{4}\left(\frac{T}{\varepsilon_{1}}\right)^{2}=\frac{S_{4}}{\pi^{2}(\zeta(3 / 2))^{4 / 3}}\left(\frac{T}{T_{c}}\right)^{2} N^{4 / 3},
$$

where the coefficient

$$
S_{4}=\sum_{\mathbf{k} \neq 0} \frac{1}{\mathbf{k}^{4}},
$$

is simply the number, $S_{4}=16.53$, and a contribution only from the first term in the Taylor expansion of the denominator in Eq. (72), $e^{\varepsilon_{1} \mathbf{k}^{2} / T}-1=\varepsilon_{1} \mathbf{k}^{2} / T+\cdots$, is important. In the box the cross-excitation parameter is not a constant, but goes to infinity in the thermodynamic limit proportionally to $N^{1 / 3}$

$$
\eta=\frac{S_{4}}{\pi^{2}(\zeta(3 / 2))^{4 / 3}}\left(\frac{T}{T_{c}}\right)^{1 / 2} N^{1 / 3} .
$$

The ratio

$$
\frac{\mathcal{H}}{\eta}=\frac{\pi^{2}(\zeta(3 / 2))^{4 / 3}}{S_{4}} \frac{T}{T_{c}} N^{2 / 3}
$$

also goes to infinity in the thermodynamic limit.

In the opposite case of very low temperatures, $T \ll \varepsilon_{1}$, we have a situation similar to that in the harmonic trap. The parameters

$$
\begin{gathered}
\mathcal{H} \approx 6 \exp \left(-\frac{\varepsilon_{1}}{T}\right) \ll 1, \\
\eta \mathcal{H} \approx 6 \exp \left(-\frac{2 \varepsilon_{1}}{T}\right) \ll 1,
\end{gathered}
$$




$$
\eta \approx \exp \left(-\frac{\varepsilon_{1}}{T}\right) \ll 1
$$

are exponentially small. The ratio

$$
\frac{\mathcal{H}}{\eta}=6
$$

is now a constant.

\section{Power-law trap}

We now consider the general case of a $d$-dimensional trap with an arbitrary power-law single-particle energy spectrum $[15,16,31]$

$$
\varepsilon_{\mathbf{k}}=\hbar \sum_{j=1}^{d} \omega_{j} k_{j}^{\sigma}, \quad \mathbf{k}=\left\{k_{j} ; j=1,2, \ldots, d\right\},
$$

where $k_{j} \geqslant 0$ is a non-negative integer and $\sigma>0$ is an index of the energy spectrum. We assume $0<\omega_{1} \leqslant \omega_{2} \leqslant \ldots$ $\leqslant \omega_{d}$, so that the energy gap between the ground state and the first excited state in the trap is $\varepsilon_{1}=\hbar \omega_{1}$. We then have

$$
\begin{aligned}
\mathcal{H} & =\sum_{\mathbf{k}>0} \frac{1}{e^{\varepsilon_{\mathbf{k}} / T}-1}, \\
\eta \mathcal{H} & =\sum_{\mathbf{k}>0} \frac{1}{\left(e^{\varepsilon_{\mathbf{k}} / T}-1\right)^{2}} .
\end{aligned}
$$

In the case $\varepsilon_{1} \ll T$, as in the box, the sum can be replaced by the integral only for the parameter $\mathcal{H}[\mathrm{Eq} .(85)]$ if $d$ $>\sigma$,

$$
\mathcal{H}=A \zeta\left(\frac{d}{\sigma}\right) T^{d / \sigma}=\left(\frac{T}{T_{c}}\right)^{d / \sigma} N, \quad d>\sigma,
$$

where the critical temperature is

$$
T_{c}=\left[\frac{N}{A \zeta(d / \sigma)}\right]^{\sigma / d}, \quad A=\frac{\left[\Gamma\left(\frac{1}{\sigma}+1\right)\right]^{d}}{\left(\prod_{j=1}^{d} \hbar \omega_{j}\right)^{1 / \sigma}} .
$$

The second parameter can be calculated by means of this continuum approximation only if $0<\sigma<d / 2$,

$$
\begin{aligned}
\eta \mathcal{H} & =A T^{d / \sigma}\left(\zeta\left(\frac{d}{\sigma}-1\right)-\zeta\left(\frac{d}{\sigma}\right)\right) \\
& =\left(\frac{T}{T_{c}}\right)^{d / \sigma} N \frac{\zeta\left(\frac{d}{\sigma}-1\right)-\zeta\left(\frac{d}{\sigma}\right)}{\zeta\left(\frac{d}{\sigma}\right)}, \quad 0<\sigma<d / 2 .
\end{aligned}
$$

If $\sigma>d / 2$, it has a formal infrared divergence and should be calculated via a discrete sum,

$$
\eta \mathcal{H}=\left(\frac{T}{T_{c}}\right)^{2} N^{2 \sigma / d} \frac{a_{\sigma, d}}{\left[\Gamma\left(\frac{1}{\sigma}+1\right)\right]^{2 \sigma}\left[\zeta\left(\frac{d}{\sigma}\right)\right]^{2 \sigma / d}}, \quad \sigma>d / 2
$$

where

$$
a_{\sigma, d}=\sum_{\mathbf{k}>0} \frac{\left(\prod_{j=1}^{d} \hbar \omega_{j}\right)^{2 / d}}{\varepsilon_{\mathbf{k}}^{2}} .
$$

The traps with a dimension lower than the critical value, $d$ $\leqslant \sigma$, can be analyzed on the basis of Eqs. (85) and (86) as well. We omit this analysis here since there is no phase transition in this case.

The cross-excitation parameter $\eta$ has different dependence on the number of atoms for high, $d>2 \sigma$, or low, $d$ $<2 \sigma$, dimensions:

$$
\begin{gathered}
\eta=\frac{\zeta\left(\frac{d}{\sigma}-1\right)-\zeta\left(\frac{d}{\sigma}\right)}{\zeta\left(\frac{d}{\sigma}\right)}, \quad d>2 \sigma>0, \\
\eta=\left(\frac{T}{T_{c}}\right)^{2-d / \sigma} N^{2 \sigma / d-1} \frac{a_{\sigma, d}}{\left[\Gamma\left(\frac{1}{\sigma}+1\right)\right]^{2 \sigma}\left[\zeta\left(\frac{d}{\sigma}\right)\right]^{2 \sigma / d}}, d<2 \sigma .
\end{gathered}
$$

Therefore the traps with small index of the energy spectrum, $0<\sigma<d / 2$, are similar to the harmonic trap (Sec. V A). The traps with larger index of the energy spectrum, $\sigma>d / 2$, are similar to the box with "homogeneous" Bose gas. For the latter traps, the cross-excitation parameter $\eta$ goes to infinity in the thermodynamic limit proportionally to $N^{2 \sigma / d-1}$. The ratio $\mathcal{H} / \eta$ goes to infinity in the thermodynamic limit only for $0<\sigma<d$. In the opposite case, $\sigma>d$, it goes to zero. We obtain

$$
\begin{gathered}
\frac{\mathcal{H}}{\eta}=\left(\frac{T}{T_{c}}\right)^{d / \sigma} N \frac{\zeta\left(\frac{d}{\sigma}\right)}{\zeta\left(\frac{d}{\sigma}-1\right)-\zeta\left(\frac{d}{\sigma}\right)}, \quad d>2 \sigma>0, \\
\frac{\mathcal{H}}{\eta}=\left(\frac{T}{T_{c}}\right)^{2(d / \sigma-1)} N^{2(1-\sigma / d)}\left[\Gamma\left(\frac{1}{\sigma}+1\right)\right]^{2 \sigma} \\
\times\left[\zeta\left(\frac{d}{\sigma}\right)\right]^{2 \sigma / d} a_{\sigma, d}^{-1}, \quad d<2 \sigma .
\end{gathered}
$$

It is remarkable that BEC occurs only for those spatial dimensions $d>\sigma$, for which $\mathcal{H} / \eta \rightarrow \infty$ at $N \rightarrow \infty$. (We do not consider here the case of the critical dimension $d=\sigma$, e.g., one-dimensional harmonic trap, where a quasi-condensation occurs at a temperature $T_{c} \sim \hbar \omega_{1} N / \ln N$.) For spatial dimensions lower than the critical value, $d<\sigma$, BEC does not oc- 

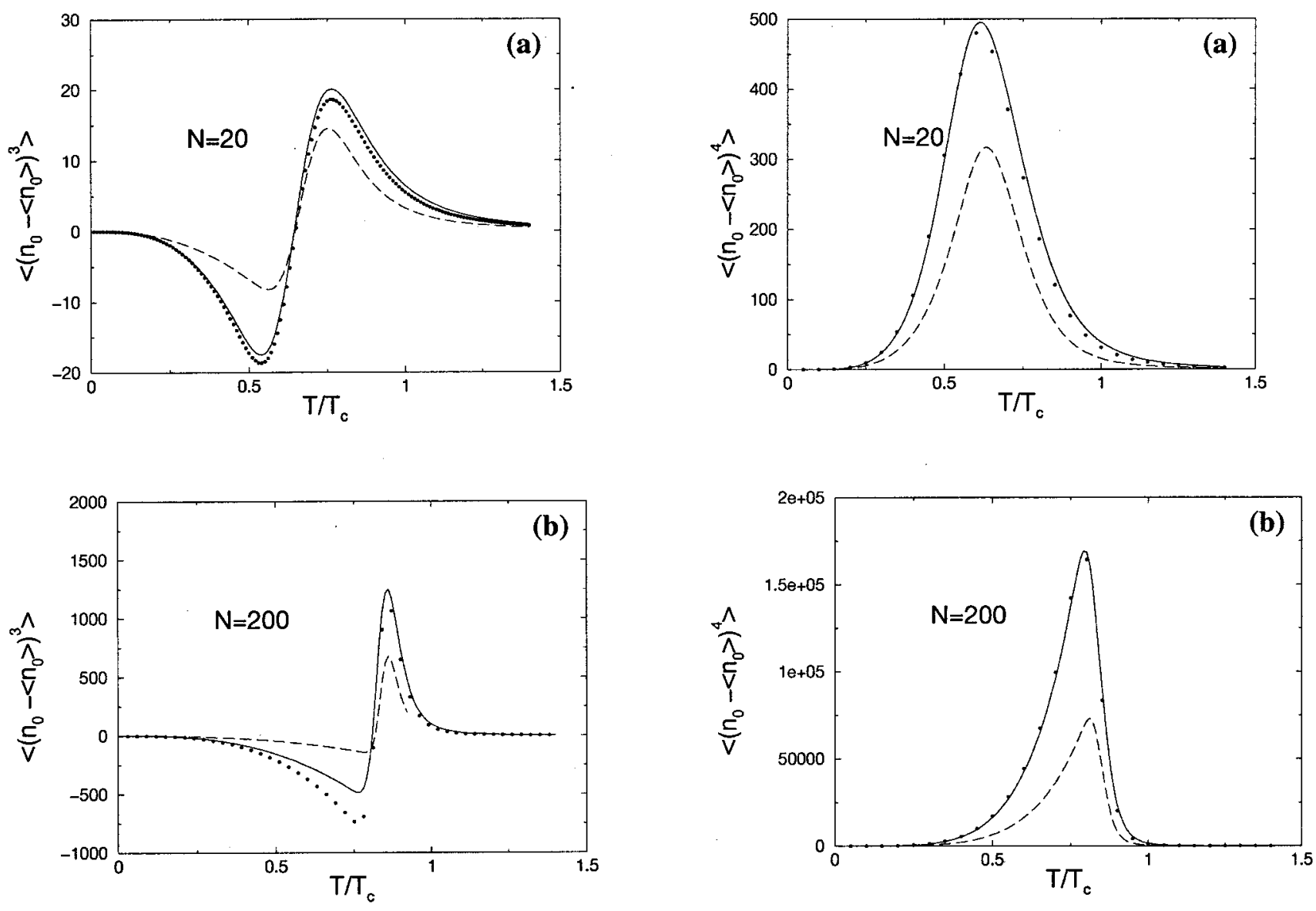

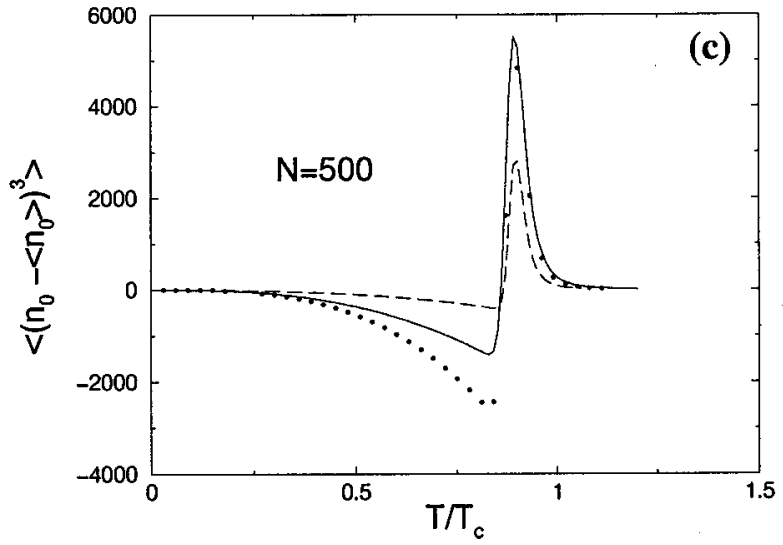

FIG. 9. The third centered moment, $\left\langle\left(n_{0}-\bar{n}_{0}\right)^{3}\right\rangle$, vs normalized temperature $T / T_{c}$, under various approximations [dashed lines for the low-temperature approximation (30), solid lines for the quasithermal approximation (40), and dots for the numerical simulation of the exact relations (44) and (46)]. Harmonic isotropic trap with (a) $N=20$, (b) $N=200$, and (c) $N=500$ atoms.

cur (see, e.g., Ref. [15]). Interestingly, even for the latter case there still exists a well-defined single peak in the probability distribution $p_{n_{0}}$ at low enough temperatures. With the help of the explicit formulas in Sec. IV, we can describe this effect as well.

In the opposite case of very low temperatures, $T \ll \varepsilon_{1}$, the parameters

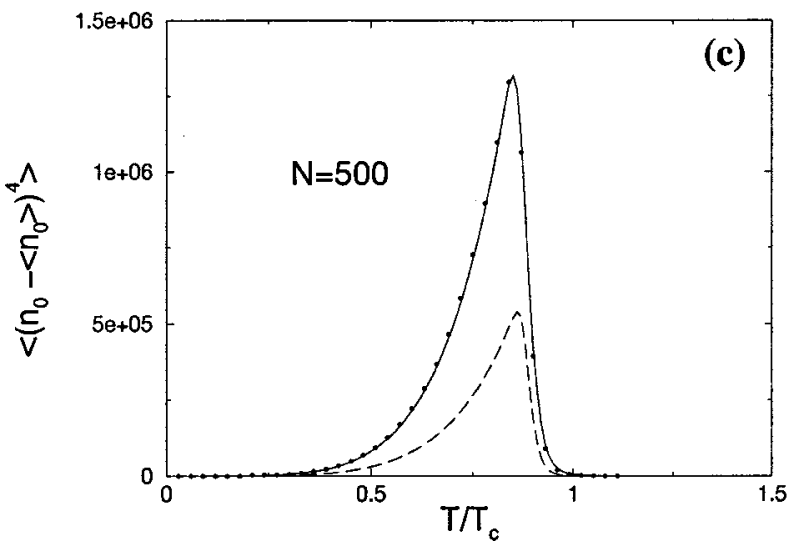

FIG. 10. The fourth centered moment, $\left\langle\left(n_{0}-\bar{n}_{0}\right)^{4}\right\rangle$, vs normalized temperature $T / T_{c}$, under various approximations [dashed lines for the low-temperature approximation (30), solid lines for the quasithermal approximation (40), and dots for the numerical simulation of the exact relations (44) and (46)]. Harmonic isotropic trap with (a) $N=20$, (b) $N=200$, and (c) $N=500$ atoms.

$$
\begin{gathered}
\mathcal{H} \approx \sum_{j=1}^{d} e^{-\hbar \omega_{j} / T}, \\
\eta \mathcal{H} \approx \sum_{j=1}^{d} e^{-2 \hbar \omega_{j} / T},
\end{gathered}
$$




$$
\eta \sim e^{-\varepsilon_{1} / T}
$$

are exponentially small. The ratio

$$
\frac{\mathcal{H}}{\eta} \sim \sum_{j=1}^{d} e^{-\left(\hbar \omega_{j}-\varepsilon_{1}\right) / T} \sim d
$$

becomes a constant.

Formulas (84)-(98) for the power-law trap contain all corresponding formulas for the three-dimensional harmonic trap $(d=3$ and $\sigma=1$; Sec. V A) and box $(d=3$ and $\sigma=2$; Sec. V B) as the particular cases.

In Figs. 4-6, we present numerical comparison of the exact results for the distribution of number of atoms in the ground level of a trap, $p_{n_{0}}$, with our approximate explicit formulas from Sec. II C in the particular case of threedimensional isotropic harmonic trap (see Sec. V A) for various temperatures. The results indicate an excellent agreement between the exact results and the results based on quasithermal approximation. We also plot the mean value $\left\langle n_{0}\right\rangle$ (Fig. 7) and the variance squared $\Delta n_{0}^{2}$ (Fig. 8) as well as the third (Fig. 9) and fourth (Fig. 10) centered moments under various approximations. In all cases our approximate results agree almost perfectly with the exact results.

\section{CONDENSATE STATISTICS IN THE THERMODYNAMIC LIMIT}

The thermodynamic, or bulk [3], limit implies an infinitely large number of atoms, $N \rightarrow \infty$, in an infinitely large trap under the condition of a fixed critical temperature, i.e., $N \omega_{x} \omega_{y} \omega_{z}=$ const in the harmonic trap, $L^{3} N=$ const in the box, and $N^{\sigma} \Pi_{j=1}^{d} \omega_{j}=$ const in an arbitrary $d$-dimensional power-law trap with an energy spectrum index $\sigma$. Then BEC takes place at the critical temperature $T_{c}$ (for $d>\sigma$ ) as a phase transition, and for some lower temperatures the factor $p_{0}$ is negligible. As a result, we have the following mean value and the variance for the number of condensed atoms:

$$
\begin{gathered}
\left\langle n_{0}\right\rangle=N-\mathcal{H} \equiv N-\sum_{k>0} \frac{1}{e^{\varepsilon_{k} / T}-1}, \\
\Delta n_{0}^{2}=(1+\eta) \mathcal{H} \equiv \sum_{k>0} \frac{1}{e^{\varepsilon_{k} / T}-1}+\sum_{k>0} \frac{1}{\left(e^{\varepsilon_{k} / T}-1\right)^{2}},
\end{gathered}
$$

which agree with the results obtained for the ideal Bose gas for different traps in the canonical ensemble by other authors $[3,15,16,30-34]$. In particular, we find the following scaling of the fluctuations of the number of condensed atoms:

$$
\begin{gathered}
\Delta n_{0}^{2} \sim C\left(\begin{array}{cc}
\left(\frac{T}{T_{c}}\right)^{d / \sigma} N, & d>2 \sigma>0 \\
\left(\frac{T}{T_{c}}\right)^{2} N^{2 \sigma / d}, & d<2 \sigma
\end{array}\right), \quad \varepsilon_{1} \ll T<T_{c}, \\
\Delta n_{0}^{2} \approx\langle n\rangle \approx \\
\sum_{i=1}^{d} \exp \left\{-\frac{\omega_{i}}{\left[\Pi_{j=1}^{d} \omega_{j}\right]^{1 / d}}\left[\zeta\left(\frac{d}{\sigma}\right)\right]^{\sigma / d}\right. \\
\\
\left.\times\left[\Gamma\left(\frac{1}{\sigma}+1\right)\right]^{\sigma} \frac{T_{c}}{T N^{\sigma / d}}\right\}, \quad T \ll \varepsilon_{1}, \quad(102)
\end{gathered}
$$

where $C$ is a constant. From Eq. (101), we see that in the high dimensional traps, $d>2 \sigma$, e.g., in the threedimensional harmonic trap, fluctuations display the proper thermodynamic behavior, $\Delta n_{0}^{2} \propto N$. However, fluctuations become anomalously large $[15,17,30], \Delta n_{0}^{2} \propto N^{2 \sigma / d} \gg N$, in the low-dimensional traps, $\sigma<d<2 \sigma$. In the quantum regime, when the temperature is less than the energy gap between the ground and first excited levels in the trap, it follows from Eq. (102) that condensate fluctuations become exponentially small. For all temperatures, when BEC exists $(d>\sigma)$, the root-mean-square fluctuations normalized to the mean number of condensed atoms vanish in the thermodynamic limit:

$$
\sqrt{\Delta n_{0}^{2}} /<n_{0}>\rightarrow 0 \quad \text { at } N \rightarrow \infty \text {. }
$$

Another remarkable property of the distribution function obtained in Sec. IV is that it yields the proper mean value and variance of the number of atoms in the ground level of the trap even for temperatures higher than the critical temperature. In particular, it can be shown that its asymptotics for high temperatures, $T \gg T_{c}$, results in a standard thermodynamic relation

$$
\Delta n_{0}^{2} \approx\left\langle n_{0}\right\rangle, \quad T \gg T_{c},
$$

that was known from the analysis of the grand-canonical ensemble [3]. This fact indicates that the present master equation approach to the statistics of the cooling Bose gas is valuable in the study of mesoscopic effects as well, both at $T<T_{c}$ and $T>T_{c}$. Note that a recently developed so-called Maxwell's Demon ensemble approach to the statistics of BEC [14-16] is valid only for temperatures well below the onset of BEC, $T<T_{c}$, and is completely wrong for $T>T_{c}$.

\section{MESOSCOPICAL AND DYNAMICAL EFFECTS IN BEC}

In recent experiments on $\mathrm{BEC}$ in ultracold gases [5], the number of condensed atoms in the trap is finite, i.e., mesoscopic rather than macroscopic, $N \sim 10^{3}-10^{6}$. Therefore it is interesting to analyze mesoscopic effects associated with the BEC statistics.

The mean number of atoms in the ground state of the trap 
with a finite number of atoms is always finite, even at high temperatures. However, it becomes macroscopically large only at temperatures lower than some critical temperature, $T_{c}$, that can be defined via the standard relation

$$
\sum_{k>0} \eta_{k}\left(T_{c}\right) \equiv \mathcal{H}\left(T_{c}\right)=N .
$$

This equation has an elementary physical meaning, that is, it determines the temperature at which the total average number of thermal excitations at all energy levels of the trap becomes equal to the total number of atoms in the trap. Results (40), (51), and (54) shown in Fig. 4-8, explicitly demonstrate a smooth transition from a mesoscopic regime (finite number of atoms in the trap, $N<\infty)$, to the thermodynamic limit $(N=\infty)$ when the threshold of the BEC becomes very sharp so that we have a phase transition to the Bose-Einstein condensed state at the critical temperature given by Eq. (105). This can be viewed as a specific demonstration of the commonly accepted resolution to the Uhlenbeck dilemma in his famous criticism of the pioneering Einstein papers on BEC [1].

Although for the systems containing a finite number of atoms there is not a sharp critical point, it is useful to define a critical characteristic value of a temperature in such a case as well. It should coincide with the standard definition (105) in the thermodynamic limit. Different definitions for $T_{c}$ were proposed and discussed in [35,12,36-42]. We follow a hint from laser physics. There we know that fluctuations dominate near threshold. However, we define a threshold inversion as that for which gain (in photon number for the lasing mode) equals loss. Let us use a similar dynamical approach for BEC on the basis of the master equation; see also Ref. [43].

We note that, for a laser operating near the threshold where $B / A \ll 1$, the equation of motion for the probability $p_{n}$ of having $n$ photons in the cavity [Eq. (1)] reduces to $[44,45]$

$$
\begin{aligned}
\frac{d p_{n}}{d t}= & -\left[A(n+1)-B(n+1)^{2}\right] p_{n}+\left[A n-B n^{2}\right] p_{n-1} \\
& -C n p_{n}+C(n+1) p_{n+1},
\end{aligned}
$$

where $A, B$, and $C$ are the linear gain, nonlinear saturation, and linear loss coefficients, respectively. Equation (106) implies the following rate of the change for the average photon number

$$
\frac{d}{d t}\langle n\rangle=(A-C)\langle n\rangle-B\left\langle(n+1)^{2}\right\rangle+A .
$$

On neglecting the spontaneous emission term $A$ and noting that the saturation term $B\left\langle(n+1)^{2}\right\rangle$ is small compared to $(A-C)\langle n\rangle$ near threshold, we define the threshold (critical) inversion to occur when the linear gain rate equals the linear loss rate, i.e., $A=C$.

Similar to laser physics, the condensate master equation (20) implies a coupled hierarchy of moment equations which are useful in the analysis of time evolution. In the quasithermal approximation (39), we find

$$
\begin{aligned}
\frac{d\left\langle n_{0}^{l}\right\rangle}{d t}= & \kappa \sum_{i=0}^{l-1}\left(\begin{array}{l}
l \\
i
\end{array}\right)\left\{( 1 + \eta ) \left[N\left(\left\langle n_{0}^{i}\right\rangle+\left\langle n_{0}^{i+1}\right\rangle\right)-\left\langle n_{0}^{i+1}\right\rangle\right.\right. \\
& \left.-\left\langle n_{0}^{i+2}\right\rangle\right]+(-1)^{l-i}(\mathcal{H}+\eta N)\left\langle n_{0}^{i+1}\right\rangle \\
& \left.-(-1)^{l-i} \eta\left\langle n_{0}^{i+2}\right\rangle\right\} .
\end{aligned}
$$

Similar moment equations in the low-temperature approximation (28) follow from Eq. (108) with $\eta=0$ :

$$
\begin{aligned}
\frac{d\left\langle n_{0}^{l}\right\rangle}{d t}= & \kappa \sum_{i=0}^{l-1}\left(\begin{array}{l}
l \\
i
\end{array}\right)\left\{N\left\langle n_{0}^{i}\right\rangle+(N-1)\left\langle n_{0}^{i+1}\right\rangle\right. \\
& \left.-\left\langle n_{0}^{i+2}\right\rangle+(-1)^{l-i} \mathcal{H}\left\langle n_{0}^{i+1}\right\rangle\right\} .
\end{aligned}
$$

The dynamical equation for the first moment, as follows from Eq. (108), has the following form:

$$
\frac{d\left\langle n_{0}\right\rangle}{d t}=\kappa\left\{(1+\eta) N+(N-1-\eta-\mathcal{H})\left\langle n_{0}\right\rangle-\left\langle n_{0}^{2}\right\rangle\right\} .
$$

Near the critical temperature $T \approx T_{c}$, the mean number of the condensed atoms is small, $\left\langle n_{0}\right\rangle \ll N$, and it is reasonable to neglect the second moment $\left\langle n_{0}^{2}\right\rangle$ compared to $N\left\langle n_{0}\right\rangle$ and the spontaneous cooling (spontaneous emission in lasers) term $\kappa N(1+\eta)$ compared to $\kappa N\left\langle n_{0}\right\rangle$. In this way, neglecting fluctuations, we arrive at a simple equation for the competition between cooling and heating processes:

$$
\frac{d\left\langle n_{0}\right\rangle}{d t} \approx \kappa(N-\mathcal{H}-\eta)\left\langle n_{0}\right\rangle .
$$

In analogy with the laser threshold we can define the critical temperature $T=T_{c}$, as a point where cooling equals heating, i.e., $d\left\langle n_{0}\right\rangle / d t=0$. This definition of the critical temperature

$$
\mathcal{H}\left(T_{c}\right)+\eta\left(T_{c}\right)=N
$$

is valid even for mesoscopic systems, and states that at $T$ $=T_{c}$ the rate of the removal of atoms from the ground state is equal to the rate of the addition, in the approximation neglecting fluctuations. In the thermodynamic limit it corresponds to the standard definition [Eq. (64) for a harmonic trap, and Eq. (75) for a box trap]. For a mesoscopic system, e.g., of $N=10^{3}$ atoms in a trap, the critical temperature as given by Eq. (112) is about $10 \%$ shifted from the thermodynamic-limit value, Eqs. (64), (75), (88). Other definitions for $T_{c}$ also describe the effect of an effective- $T_{c}$ shift $[35,12,36-42]$ and agree qualitatively with our definition.

Note that precisely the same definition of the critical temperature follows from a statistical mechanical point of view, which in some sense is alternative to the dynamical one. We 
may define the critical temperature as the temperature at which the mean number of condensed atoms in the steadystate solution to the master equation vanishes when neglecting fluctuations and spontaneous cooling. We make the replacement $\left\langle n_{0}^{2}\right\rangle \approx\left\langle n_{0}\right\rangle^{2}$ in Eq. (110) and obtain the steadystate solution to this nonlinear equation, $\left\langle n_{0}\right\rangle=N-\mathcal{H}-\eta$. Now we see that $\left\langle n_{0}\right\rangle$ vanishes at the same critical temperature (112).

\section{CONCLUSIONS}

It is interesting to note that the first results for the average and variance of occupation numbers in the ideal Bose gas in the canonical ensemble were obtained about 50 years ago by the standard statistical methods [32,33] (see also Refs. $[30,34]$ and review [3]). Only later, in the 1960s, was the laser physics and its byproduct, the master equation approach, developed (see, e.g., Refs. [10,45]). In this paper we have shown that the latter approach provides very simple and effective tools to calculate statistical properties of the ideal Bose gas cooling by the thermal reservoir. In particular, our results (51) and (54) reduce to the mentioned old results in the "condensed region" $N-N_{c} \gg N_{c} \sqrt{T / \varepsilon_{1}}$ in the thermodynamic limit.

However, the master equation approach gives even more. It yields simple analytical expressions for the distribution function of the number of condensed atoms [Eq. (40)] and for the canonical partition function (41). In terms of cumulants, or semi-invariants, [29] for the stochastic variables $n_{0}$ or $n=N-n_{0}$, we can show [18] that the quasithermal approximation (35), with results (51) and (54), gives correctly both the first and second cumulants. The analysis of the higher-order cumulants is more complicated, and includes in principle a comparison with more accurate calculations of the conditioned average number of noncondensed atoms [Eq. (19)] as well as higher-order corrections to the second order master equation (12). It is therefore clear that the master equation approach is capable of giving the correct answer for all higher-order cumulants and, therefore, moments of the condensate fluctuations. In the present paper, we demonstrated that even without these complications our approximate result (40) reproduces the higher moments, calculated numerically via the exact recursion relation (46), remarkably well for all temperatures $T<T_{c}$ and $T \sim T_{c}$ (see Figs. 8-10).

As we demonstrated in Secs. II and V, the simple formulas yielded by the master equation approach allow us to study mesoscopic effects in BEC for a relatively small number of atoms that is typical for recent experiments [5]. Moreover, it is interesting in the study of the dynamics of BEC (Sec. VII). This technique for studying statistics and dynamics of BEC shows surprisingly good results even within the simplest approximations. Thus the analogy with phase transitions and quantum fluctuations in lasers (see, e.g., Refs. $[10,11,21,22])$ clarifies some problems in BEC. The present paper is largely devoted to the equilibrium properties of the number-of-condensed-atom statistics which are relatively insensitive to the details of the model. The origin of dynamical and coherent properties of the evaporatively cooling gas with an interatomic interaction is conceptually different from that in the present "ideal gas plus thermal reservoir" model. The present model is rather close to the dilute ${ }^{4} \mathrm{He}$ gas in porous gel experiments [4] in which phonons in the gel play the role of the external thermal reservoir. Nevertheless, the noncondensed atoms always play a part of some internal reservoir and the condensate master equation probably contains terms similar to those in Eq. (20) for any cooling mechanism.

The problem of dynamics and fluctuations of BEC for the interacting gas is much more involved. The master equation approach provides a very powerful tool for the solution of this problem as well. Of course, to take into account higherorder effects of interaction between atoms, we have to go beyond the second-order master equation, i.e., to iterate Eq. (11) more times and to proceed with the higher-order master equation similarly to that we discussed above. It is possible to show [46] that the master equation approach allows us to take into account all higher order effects in a way generalizing a well-known nonequilibrium Keldysh diagram technique [47-49]. As a result, the second-order master equation analysis presented above can be justified rigorously, and higher-order effects in condensate fluctuations at equilibrium as well as nonequilibrium stages of cooling of both ideal and interacting Bose gases can be calculated. These aspects of the problem will be discussed elsewhere.

We mention here only an important result of an analytical calculation of all higher cumulants (moments) [18]. In most cases (except for the ideal gas in the harmonic trap and similar high-dimensional traps where $d>26$ ), both for the ideal Bose gas and for the interacting Bose gas, the third and higher cumulants of the number-of-condensed-atom fluctuations normalized to the corresponding power of the variance, e.g., $\left\langle\left(n_{0}-\left\langle n_{0}\right\rangle\right)^{3}\right\rangle /\left\langle\left(n_{0}-\left\langle n_{0}\right\rangle\right)^{2}\right\rangle^{3 / 2}$, do not vanish in the thermodynamic limit. Thus fluctuations in BEC are not Gaussian contrary to what is usually assumed. They are, in fact, anomalously large and constitute an important open problem in the physics of many-body systems.

\section{ACKNOWLEDGMENTS}

We thank Kishore Kapale for numerical simulations and for preparing the figures. We also thank G. Baym, S. A. Chin, R. Glauber, M. Girardeau, M. Holthaus, C. R. Hu, K. Huang, R. Hulet, W. Ketterle, D. Kleppner, W. E. Lamb, P. Lee, P. Meystre, J. Phillips, V. Pokrovsky, M. Wilkens, and C. N. Yang for helpful discussions. One of us (M.S.Z.) would like to thank W. Schleich for his hospitality at Universität Ulm, where part of this work was carried out. This work was supported by the Office of Naval Research, The National Science Foundation and the Welch Foundation.

\section{APPENDIX A: DERIVATION OF THE MASTER EQUATION (12)}

It follows, on substituting the expression for $V$ from Eq. (9) into Eq. (11), that 


$$
\begin{aligned}
& \dot{\rho}(t)=-\frac{1}{\hbar^{2}} \int_{0}^{t} d t^{\prime} \operatorname{Tr}_{\text {res }}\left[V(t) V\left(t^{\prime}\right) \rho_{\text {total }}\left(t^{\prime}\right)-V(t) \rho_{\text {total }}\left(t^{\prime}\right) V\left(t^{\prime}\right)+\text { H.c. }\right] \\
& =-\frac{g^{2}}{\hbar^{2}} \int_{0}^{t} d t^{\prime} \operatorname{Tr}_{\mathrm{res}}\left[\sum _ { j , j ^ { \prime } } \sum _ { k > l , k ^ { \prime } > l ^ { \prime } } \left[b_{j}^{\dagger} b_{j^{\prime}} a_{k} a_{l}^{\dagger} a_{l^{\prime}} a_{k^{\prime}}^{\dagger} e^{-i\left(\omega_{j}-\omega_{k l}\right) t} e^{i\left(\omega_{j^{\prime}}-\omega_{k^{\prime} l^{\prime}}\right) t^{\prime}}\right.\right. \\
& \left.+b_{j} b_{j^{\prime}}^{\dagger} a_{l} a_{k}^{\dagger} a_{k^{\prime}} a_{l^{\prime}}^{\dagger} e^{i\left(\omega_{j}-\omega_{k l}\right) t} e^{-i\left(\omega_{j^{\prime}}-\omega_{k^{\prime} l^{\prime}}\right) t^{\prime}}\right] \rho_{\text {total }}\left(t^{\prime}\right) \\
& -\sum_{j, j^{\prime}} \sum_{k>l, k^{\prime}>l^{\prime}} b_{j}^{\dagger} a_{k} a_{l}^{\dagger} \rho_{\text {total }}\left(t^{\prime}\right) b_{j^{\prime}} a_{l^{\prime}} a_{k^{\prime}}^{\dagger} e^{-i\left(\omega_{j}-\omega_{k l}\right) t} e^{i\left(\omega_{j^{\prime}}-\omega_{k^{\prime} l^{\prime}}\right) t^{\prime}} \\
& +b_{j} a_{l} a_{k}^{\dagger} \rho_{\text {total }}\left(t^{\prime}\right) b_{j^{\prime}}^{\dagger} a_{k^{\prime}} a_{l^{\prime}}^{\dagger} e^{i\left(\omega_{j}-\omega_{k l}\right) t} e^{-i\left(\omega_{j^{\prime}}-\omega_{\left.k^{\prime} l^{\prime}\right) t^{\prime}}+\text { H.c. }\right]} \\
& =-\frac{g^{2}}{\hbar^{2}} \int_{0}^{t} d t^{\prime}\left(\sum _ { k > l , k ^ { \prime } > l ^ { \prime } } \operatorname { T r } _ { \mathrm { res } } \left[\sum _ { j } \left(b_{j}^{\dagger} b_{j} \rho_{\mathrm{res}} a_{k} a_{l}^{\dagger} a_{l^{\prime}} a_{k^{\prime}}^{\dagger} e^{-i\left(\omega_{j}-\omega_{k^{\prime} l^{\prime}}\right)\left(t-t^{\prime}\right)} e^{i\left(\omega_{k l}-\omega_{k^{\prime} l^{\prime}}\right) t} \rho\left(t^{\prime}\right)\right.\right.\right. \\
& \left.+b_{j} b_{j}^{\dagger} \rho_{\mathrm{res}} a_{l} a_{k}^{\dagger} a_{k^{\prime}} a_{l^{\prime}}^{\dagger} e^{i\left(\omega_{j}-\omega_{k^{\prime} l^{\prime}}\right)\left(t-t^{\prime}\right)} e^{-i\left(\omega_{k l}-\omega_{k^{\prime} l^{\prime}}\right) t} \rho\left(t^{\prime}\right)\right) \\
& -\sum_{j}\left(b_{j}^{\dagger} \rho_{\mathrm{res}} b_{j} a_{k} a_{l}^{\dagger} \rho\left(t^{\prime}\right) a_{l^{\prime}} a_{k^{\prime}}^{\dagger} e^{-i\left(\omega_{j}-\omega_{k^{\prime} l^{\prime}}\right)\left(t-t^{\prime}\right)} e^{i\left(\omega_{k l}-\omega_{k^{\prime} l^{\prime}}\right) t}\right. \\
& \left.\left.\left.+b_{j} \rho_{\text {res }} b_{j}^{\dagger} a_{l} a_{k}^{\dagger} \rho\left(t^{\prime}\right) a_{k^{\prime}} a_{l^{\prime}}^{\dagger} e^{i\left(\omega_{j}-\omega_{k^{\prime} l^{\prime}}\right)\left(t-t^{\prime}\right)} e^{-i\left(\omega_{k l}-\omega_{k^{\prime} l^{\prime}}\right) t}\right)+ \text { H.c. }\right]\right),
\end{aligned}
$$

where we assumed that the reservoir has almost no change with the interaction with the system so that we can write $\rho_{\text {total }}\left(t^{\prime}\right)=\rho_{\text {res }} \otimes \rho\left(t^{\prime}\right)$, with $\rho_{\text {res }}$ being independent of time. We also used $\left\langle b_{j} b_{j^{\prime}}\right\rangle_{\text {res }}=\left\langle b_{j}^{\dagger} b_{j^{\prime}}^{\dagger}\right\rangle_{\text {res }}=0$. In thermal equilibrium at temperature $T,\left\langle b_{j} b_{j^{\prime}}^{\dagger}\right\rangle_{\text {res }}=\left[1+\eta\left(\omega_{j}\right)\right] \delta_{j, j^{\prime}}$ and $\left\langle b_{j}^{\dagger} b_{j^{\prime}}\right\rangle_{\text {res }}=\eta\left(\omega_{j}\right) \delta_{j, j^{\prime}}$, where $\eta\left(\omega_{j}\right)$ is the average phonon number of the reservoir at the frequency $\omega_{j}$ and is given by Eq. (13). On replacing the summation over $\mathrm{j}$ by an integration and carrying out the integration, we have

$$
\begin{aligned}
& \dot{\rho}(t)=-\pi \frac{g^{2}}{\hbar^{2}} D \sum_{k>l, k^{\prime}>l^{\prime}}\left(\eta\left(\omega_{k^{\prime} l^{\prime}}\right) e^{i\left(\omega_{k l}-\omega_{k^{\prime} l^{\prime}}\right) t} a_{k} a_{l}^{\dagger} a_{l^{\prime}} a_{k^{\prime}}^{\dagger} \rho(t)\right. \\
& +\left[\eta\left(\omega_{k^{\prime} l^{\prime}}\right)+1\right] e^{-i\left(\omega_{k l}-\omega_{k^{\prime} l^{\prime}}\right) t} a_{l} a_{k}^{\dagger} a_{k^{\prime}} a_{l^{\prime}}^{\dagger} \rho(t)-\left[\eta\left(\omega_{k^{\prime} l^{\prime}}\right)+1\right] \\
& \left.\times e^{i\left(\omega_{k l}-\omega_{k^{\prime} l^{\prime}}\right) t} a_{k} a_{l}^{\dagger} \rho(t) a_{l^{\prime}} a_{k^{\prime}}^{\dagger}-\eta\left(\omega_{k^{\prime} l^{\prime}}\right) e^{-i\left(\omega_{k l}-\omega_{k^{\prime} l^{\prime}}\right) t} a_{l} a_{k}^{\dagger} \rho(t) a_{k^{\prime}} a_{l^{\prime}}^{\dagger}+\text { H.c. }\right) \text {, }
\end{aligned}
$$

where it was assumed that the mode density for the reservoir $D\left(\omega_{k^{\prime} l^{\prime}}\right)$ varies very slowly with frequency and can be considered as a constant $D$. The terms containing the factor $e^{i\left(\omega_{k l}-\omega_{k^{\prime} l^{\prime}}\right)}$ with $\omega_{k l} \neq \omega_{k^{\prime} l^{\prime}}$ will average out in the double summations. We therefore retain only those terms with $\omega_{k l}=\omega_{k^{\prime} l^{\prime}}$ in the above equation after taking out one summation. If we define $\kappa=2 \pi D g^{2} / \hbar^{2}$, we obtain Eq. (12).

\section{APPENDIX B: DERIVATION OF EXPRESSIONS FOR $\boldsymbol{\eta} \mathcal{H}$ FOR DIFFERENT TRAPS}

(a) For the harmonic potential,

$$
\eta \mathcal{H}=\sum_{\mathbf{k}>0} \frac{1}{\left(e^{\hbar \mathbf{k} \boldsymbol{\omega} / T}-1\right)^{2}} .
$$

For $\varepsilon_{1} \ll T$, the sum can be replaced by an integral, and we obtain 


$$
\begin{aligned}
\eta \mathcal{H} \simeq & \int_{0}^{\infty} d k_{z} \int_{0}^{\infty} \frac{d k_{y}}{e^{2 \hbar\left(\omega_{y} k_{y}+\omega_{z} k_{z}\right) / T}} \\
& \times \int_{0}^{\infty} \frac{d k_{x}}{\left(e^{\hbar \omega_{x} k_{x} / T}-e^{-\hbar\left(\omega_{y} k_{y}+\omega_{z} k_{z}\right) / T}\right)^{2}} \\
= & \frac{T}{\hbar \omega_{x}} \sum_{k=0}^{\infty} \frac{k+1}{k+2} \int_{0}^{\infty} d k_{z} \int_{0}^{\infty} d k_{y} e^{-(k+2) \hbar\left(\omega_{y} k_{y}+\omega_{z} k_{z}\right) / T} \\
= & \frac{T^{3}}{\hbar^{3} \omega_{x} \omega_{y} \omega_{z}} \sum_{k=0}^{\infty}\left(\frac{1}{(k+2)^{2}}-\frac{1}{(k+2)^{3}}\right) .
\end{aligned}
$$

We thus have

$$
\eta \mathcal{H}=\frac{T^{3}}{\hbar^{3} \omega_{x} \omega_{y} \omega_{z}}(\zeta(2)-\zeta(3)),
$$

where

$$
\zeta(i)=\sum_{k=0}^{\infty} \frac{1}{(k+2)^{i}} .
$$

(b) For the box potential,

$$
\eta \mathcal{H}=\sum_{\mathbf{k} \neq 0} \frac{1}{\left(e^{\varepsilon_{1} \mathbf{k}^{2} / T}-1\right)^{2}},
$$

where

$$
\varepsilon_{1}=\frac{2 \pi^{2} \hbar^{2}}{m L^{2}}
$$

is the energy gap between the ground state and the first excited state in the box. In the case $\varepsilon_{1} \ll T$ we cannot make the continuum approximation. However, we retain only the leading term in the summation, i.e.,

$$
\eta \mathcal{H} \simeq \sum_{\mathbf{k} \neq 0} \frac{T^{2}}{\varepsilon_{1}^{2} \mathbf{k}^{4}}=\left(\frac{T}{\varepsilon_{1}}\right)^{2} S_{4},
$$

where

$$
S_{4}=\sum_{\mathbf{k} \neq 0} \frac{1}{\mathbf{k}^{4}}=16.53 .
$$

(c) In the power law trap [Eq. (84)] we have two cases: $0<\sigma<d / 2$ and $\sigma>d / 2$. For $0<\sigma<d / 2$, the expression for $\eta \mathcal{H}$ is derived as in (a) above and is given by Eq. (89), whereas, for $\sigma>d / 2$, we retain only the leading term in the summation as in (b) above and obtain Eq. (90), derived as in (b) above.

In particular we note that

$$
\begin{gathered}
\int_{0}^{\infty} \cdots \int_{0}^{\infty} f\left(k_{1}^{\sigma}+\cdots+k_{d}^{\sigma}\right) d k_{1} \cdots d k_{d} \\
=\frac{\left[\Gamma\left(\frac{1}{\sigma}\right)\right]^{d}}{\Gamma\left(\frac{d}{\sigma}\right)} \int_{0}^{\infty} f(t) t^{(d / \sigma)-1} d t .
\end{gathered}
$$

Also we have, for $f(t)=1 /\left(e^{t}-1\right)$,

$$
\int_{0}^{\infty} \frac{t^{(d / \sigma)-1}}{e^{t}-1} d t=\Gamma\left(\frac{d}{\sigma}\right) \zeta\left(\frac{d}{\sigma}\right)
$$

and, for $f(t)=1 /\left(e^{t}-1\right)^{2}$,

$$
\int_{0}^{\infty} \frac{t^{(d / \sigma)-1}}{\left(e^{t}-1\right)^{2}} d t=\Gamma\left(\frac{d}{\sigma}\right)\left[\zeta\left(\frac{d}{\sigma}-1\right)-\zeta\left(\frac{d}{\sigma}\right)\right] .
$$

These equations readily yield Eqs. (87) and (89).
[1] S. N. Bose, Z. Phys. 26, 178 (1924); A. Einstein, Berl. Ber. 22, 261 (1924); ibid. 23, 3 (1925); ibid. 23, 18 (1925); G. E. Uhlenbeck, Ph.D. thesis, Leiden, 1927; B. Kahn and G. E. Uhlenbeck, Physica (Amsterdam) 5, 399 (1938); F. London, Phys. Rev. 54, 947 (1938); J. Chem. Phys. 48, 49 (1939); ibid. 11, 203 (1943); Superfluids II (Dover, New York, 1954).

[2] The classic works in interacting Bose gas physics by Bogoliubov, Huang, Lee, and Yang are well presented by K. Huang, Statistical Mechanics (Wiley, New York 1987), Chap. 19; M. Girardeau and R. Arnowitt, Phys. Rev. 113, 755 (1959); A. Griffin, Excitations in a Bose-Condensed Liquid (Cambridge University Press, Cambridge 1993); Bose Einstein Condensation, edited by A. Griffin, D. Snoke, and G. Stringari (Cambridge University Press, Cambridge, 1995); G. Baym and C. Pethick, Phys. Rev. Lett. 76, 6 (1996).
[3] R. M. Ziff, G. E. Uhlenbeck, and M. Kac, Phys. Rep. 32, 169 (1977).

[4] B. C. Crooker, B. Hebral, E. N. Smith, Y. Takano, and J. D. Reppy, Phys. Rev. Lett. 51, 666 (1983); M. H. W. Chan, K. I. Blum, S. Q. Murphy, G. K. S. Wong, and J. D. Reppy, ibid. 61, 1950 (1988); P. A. Crowell, F. W. Van Keuls, and J. D. Reppy, ibid. 75, 1106 (1995).

[5] M. Anderson, J. Ensher, M. Matthews, C. Wieman, and E. Cornell, Science 269, 198 (1995); C. Bradley, C. Sackett, J. Tollett, and R. Hulet, Phys. Rev. Lett. 75, 1687 (1995); K. Davis, M. Mewes, M. Andrews, N. van Druten, D. Durfee, D. Kurn, and W. Ketterle, ibid. 75, 3969 (1995). Independent observations of BEC were made by D. J. Han, R. H. Wynar, Ph. Courteille, and D. J. Heinzen, Phys. Rev. A 57, R4114 (1998); U. Ernst, A. Marte, F. Schreck, J. Schuster, and G. Rempe, 
Europhys. Lett. 41, 1 (1998); L. V. Hau, B. D. Busch, C. Liu, Z. Dutton, M. M. Burns, and J. A. Golovchenko, Phys. Rev. A 58, R54 (1998); T. Esslinger, I. Bloch, and T. W. Hänsch, ibid. 58, R2664 (1998); B. P. Anderson and M. A. Kasevich, ibid. 59, R938 (1999).

[6] BEC in atomic hydrogen was observed by D. G. Fried, T. C. Killian, L. Willmann, D. Landhuis, S. C. Moss, D. Kleppner, and T. J. Greytak, Phys. Rev. Lett. 81, 3811 (1998).

[7] For laser cooling techniques that were used in recent BEC experiments, see S. Chu, Rev. Mod. Phys. 70, 685 (1998); C. N. Cohen-Tannoudji, ibid. 70, 707 (1998); W. D. Phillips, ibid. 70, 721 (1998).

[8] H.-J. Miesner, D. Stamper-Kurn, M. Andrews, D. Durfee, S. Inouye, and W. Ketterle, Science 279, 1005 (1998).

[9] M.-O. Mewes, M. R. Andrews, D. M. Kurn, D. S. Durfee, C. G. Townsend, and W. Ketterle, Phys. Rev. Lett. 78, 582 (1997); M. R. Andrews, C. G. Townsend, H.-J. Miesner, D. S. Durfee, D. M. Kurn, and W. Ketterle, Science 275, 637 (1997); B. P. Anderson and M. A. Kasevich, ibid. 282, 1686 (1998); I. Bloch, T. W. Hansch, and T. Esslinger, Phys. Rev. Lett. 82, 3008 (1999).

[10] M. O. Scully and W. E. Lamb, Jr., Phys. Rev. Lett. 16, 853 (1966) developed the quantum theory of laser in the present density-matrix form. The quantum theory of lasers as developed independently by Haken and co-workers and Lax and co-workers is given in M. O. Scully and M. S. Zubairy, Quantum Optics (Cambridge University Press, Cambridge 1997).

[11] M. O. Scully, Phys. Rev. Lett. 82, 3927 (1999); henceforth referred to as CNB I.

[12] W. Ketterle and N. van Druten, Phys. Rev. A 54, 656 (1996).

[13] M. Holthaus, E. Kalinowski, and K. Kristen, Ann. Phys. (N.Y.) 270, 198 (1998).

[14] H. Politzer, Phys. Rev. A 54, 5048 (1996). See also S. Grossmann and M. Holthaus, Phys. Rev. E 54, 3495 (1996); P. Navez, D. Bitouk, M. Gajda, Z. Idziaszek, and K. Rzazewski, Phys. Rev. Lett. 79, 1789 (1997); N. L. Balazs and T. Bergeman, Phys. Rev. A 58, 2359 (1998).

[15] M. Wilkens and C. Weiss, J. Mod. Opt. 44, 1801 (1997); C. Weiss and M. Wilkens, Opt. Express 1, 272 (1997).

[16] S. Grossmann and M. Holthaus, Phys. Rev. Lett. 79, 3557 (1997).

[17] S. Giorgini, L. P. Pitaevskii, and S. Stringari, Phys. Rev. Lett. 80, 5040 (1998).

[18] V. V. Kocharovsky, Vl. V. Kocharovsky, and M. O. Scully (unpublished).

[19] C. Kittel, Elementary Statistical Physics (Wiley, New York 1958), p. 62.

[20] C. Herzog and M. Olshanii, Phys. Rev. A 55, 3254 (1997).

[21] V. DeGiorgio and M. O. Scully, Phys. Rev. A 2, 1170 (1970).

[22] R. Graham and H. Haken, Z. Phys. 237, 31 (1970).
[23] M. Gajda and K. Rzazewski, Phys. Rev. Lett. 78, 2686 (1997).

[24] P. T. Landsberg, Thermodynamics—with Quantum Statistical Illustrations (Interscience, New York 1961).

[25] F. C. Auluck and D. S. Kothari, Proc. Cambridge Philos. Soc. 42, 272 (1946).

[26] M. Toda, R. Kubo, and N. Saito, Statistical Physics I (Springer-Verlag, Berlin, 1992).

[27] P. Borrmann and G. Franke, J. Chem. Phys. 98, 2484 (1993).

[28] F. Brosens, J. J. Devreese, and L. F. Lemmens, Solid State Commun. 100, 123 (1996).

[29] Handbook of Mathematical Functions, edited by M. Abramowitz and I. A. Stegun (Dover, New York, 1972), Chap. 26.

[30] E. H. Hauge, Phys. Norv. 4, 19 (1969).

[31] S. R. de Groot, G. J. Hooyman, and C. A. ten Seldam, Proc. R. Soc. London, Ser. A 203, 266 (1950).

[32] R. B. Dingle, Proc. Cambridge Philos. Soc. 45, 275 (1949); Adv. Phys. 1, 117 (1952); Asymptotic Expansions (Academic Press, New York, 1973), pp. 267-271.

[33] A. R. Fraser, Philos. Mag. 42, 165 (1951).

[34] F. Reif, Fundamentals in Thermal Physics (McGraw-Hill, New York, 1965).

[35] S. Grossmann and M. Holthaus, Z. Naturforsch., A: Phys. Sci. 50a, 921 (1995).

[36] S. Giorgini, L. P. Pitaevskii, and S. Stringari, Phys. Rev. A 54, R4633 (1996).

[37] K. Kirsten and D. J. Toms, Phys. Rev. A 54, 4188 (1996); 59, 158 (1999).

[38] H. Haugerud, T. Haugest, and F. Ravndal, Phys. Lett. A 225, 18 (1997).

[39] M. Bergaman, Phys. Rev. A 55, 3658 (1997); 56, 3310 (1997).

[40] M. Houbiers, H. T. C. Stoof, and E. A. Cornell, Phys. Rev. A 56, 2041 (1997).

[41] P. Gruter, D. Ceperley, and F. Laloe, Phys. Rev. Lett. 79, 3553 (1997).

[42] R. K. Pathria, Phys. Rev. A 58, 1490 (1998).

[43] M. Scully and K. Kapale, in Proceedings of the R. Arnowitt Fest: Relativity, Particle Physics and Cosmology, edited by R. E. Allen (World Scientific, Singapore, 1999), pp. 181-189.

[44] M. Sargent III, M. O. Scully, and W. E. Lamb, Jr., Appl. Opt. 9, 2423 (1970).

[45] M. Sargent III, M. Scully, and W. E. Lamb, Jr., Laser Physics (Addison-Wesley, Reading, MA, 1974).

[46] V. V. Kocharovsky, Vl. V. Kocharovsky, and M. O. Scully (unpublished).

[47] L. V. Keldysh, Zh. Éksp. Teor. Fiz. 47, 352 (1964) [Sov. Phys. JETP 20, 234 (1965)].

[48] E. M. Lifshitz and L. P. Pitaevskii, Statistical Physics (Pergamon, Oxford, 1981), pt. 2.

[49] H. T. C. Stoof, J. Low Temp. Phys. 114, 11 (1999). 